\title{
Regularity of the free boundary in the biharmonic obstacle problem
}

\author{
Gohar Aleksanyan ${ }^{1}$
}

Received: 22 March 2016 / Accepted: 30 September 2019 / Published online: 7 November 2019

(c) The Author(s) 2019

\begin{abstract}
In this article we use a flatness improvement argument to study the regularity of the free boundary for the biharmonic obstacle problem with zero obstacle. Assuming that the solution is almost one-dimensional, and that the non-coincidence set is an non-tangentially accessible domain, we derive the $C^{1, \alpha}$-regularity of the free boundary in a small ball centred at the origin. From the $C^{1, \alpha}$-regularity of the free boundary we conclude that the solution to the biharmonic obstacle problem is locally $C^{3, \alpha}$ up to the free boundary, and therefore $C^{2,1}$. In the end we study an example, showing that in general $C^{2, \frac{1}{2}}$ is the best regularity that a solution may achieve in dimension $n \geq 2$.
\end{abstract}

Mathematics Subject Classification Primary 35R35; Secondary 35J35 · 31 B30

\section{Contents}

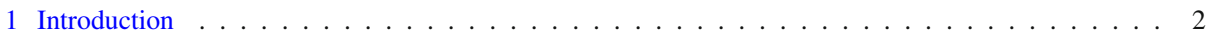

2 The obstacle problem for the biharmonic operator . . . . . . . . . . . . . . 3

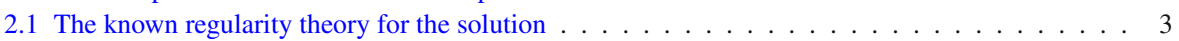

3 Regularity of the free boundary . . . . . . . . . . . . . . . . . . . . . . . . . 6

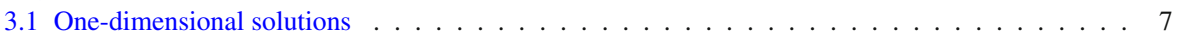

3.2 The class $\mathscr{B}_{\kappa}^{\varrho}(\varepsilon)$ of solutions to the biharmonic obstacle problem . . . . . . . . . . . 8

3.3 Linearisation . . . . . . . . . . . . . . . . . . . . . . . . . . . . . 12

3.4 Properties of solutions in a normalised coordinate system . . . . . . . . . . . . . . . . . . . 14

$3.5 C^{1, \alpha}$-regularity of the free boundary . . . . . . . . . . . . . . . . . . . . 20

4 On the regularity of the solution . . . . . . . . . . . . . . . . . . . . . . . . . . . 24

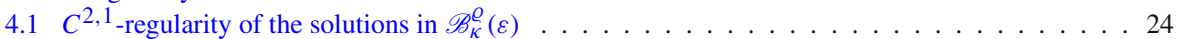

4.2 In general the solutions are not better than $C^{2, \frac{1}{2}} \ldots \ldots \ldots \ldots \ldots \ldots$

A Estimates on derivatives of biharmonic functions . . . . . . . . . . . . . . . . . . . . 26

References . . . . . . . . . . . . . . . . . . . . . . . . 28

Communicated by O. Savin.

Gohar Aleksanyan

galeksan@kth.se

1 KTH Royal Institute of Technology, Stockholm, Sweden 


\section{Introduction}

Let $\Omega \subset \mathbb{R}^{n}$ be a given domain, and $\varphi \in C^{2}(\bar{\Omega}), \varphi \leq 0$ on $\partial \Omega$ be a given function, called an obstacle. Then the minimiser to the following functional

$$
J[u]=\int_{\Omega}(\Delta u(x))^{2} d x,
$$

over all functions $u \in W_{0}^{2,2}(\Omega)$, such that $u \geq \varphi$, is called the solution to the biharmonic obstacle problem with obstacle $\varphi$. The solution satisfies the following variational inequalities

$$
\Delta^{2} u \geq 0, u \geq \varphi, \Delta^{2} u \cdot(u-\varphi)=0 .
$$

It has been shown in [1] that the solution $u \in W_{l o c}^{3,2}(\Omega)$, and in [2] that $\Delta u \in L_{l o c}^{\infty}(\Omega)$, and moreover $u \in W_{l o c}^{2, \infty}(\Omega)$, see also [3]. Furthermore, in the paper [3], the authors show that in dimension $n=2$ the solution $u \in C^{2}(\Omega)$ and that the free boundary $\Gamma_{u}:=\partial\{u=\varphi\}$ lies on a $C^{1}$-curve in a neighbourhood of the points $x_{0} \in \Gamma_{u}$, such that $\Delta u\left(x_{0}\right)>\Delta \varphi\left(x_{0}\right)$.

The setting of our problem is slightly different from the one in [1,3] and [2]. We consider a zero-obstacle problem with general nonzero boundary conditions. Let $\Omega$ be a bounded domain in $\mathbb{R}^{n}$ with smooth boundary. We study the minimiser of the functional (1.1) over the admissible set

$$
\mathscr{A}:=\left\{u \in W^{2,2}(\Omega), u \geq 0, u=g>0, \frac{\partial u}{\partial v}=f \text { on } \partial \Omega\right\} .
$$

The minimiser $u$ exists, it is unique, and is called the solution to the biharmonic obstacle problem. We will denote the free boundary by $\Gamma_{u}:=\partial \Omega_{u} \cap \Omega$, where $\Omega_{u}:=\{u>0\}$.

There are several important questions regarding the biharmonic obstacle problem that remain open. For example, the optimal regularity of the solution, the characterisation of blow-ups at free boundary points, etc. In this article we focus on the regularity of the free boundary for an $n$-dimensional biharmonic obstacle problem, assuming that the solution is close to the one-dimensional solution $\frac{1}{6}\left(x_{n}\right)_{+}^{3}$. In [4], using flatness improvement argument, the author, John Andersson, shows that the free boundary in the $p$-harmonic obstacle problem is a $C^{1, \alpha}$ graph in a neighbourhood of the points where the solution is almost one-dimensional. We apply the same technique in order to study the regularity of the free boundary in the biharmonic obstacle problem.

In Sect. 2 we study the basic properties of the solution, adapted to our setting. The existence, uniqueness as well as $W_{l o c}^{3,2} \cap C_{l o c}^{1, \alpha}$-regularity of the solution are known. For the sake of completeness statements and some sketched proofs are included, together with references.

In Sect. 3 we introduce the class $\mathscr{B}_{K}^{\varrho}(\varepsilon)$ of solutions to the biharmonic obstacle problem, which are close to the one-dimensional solution $\frac{1}{6}\left(x_{n}\right)_{+}^{3}$. Following the linearisation argument by John Andersson (see for instance [4]), we show that if $\varepsilon$ is small enough, then there exists a rescaling $u_{s}(x)=\frac{u(s x)}{s^{3}}$, such that

$$
\left\|\nabla^{\prime} u_{s}\right\|_{W^{2,2}\left(B_{2}\right)} \leq \gamma\left\|\nabla^{\prime} u\right\|_{W^{2,2}\left(B_{2}\right)} \leq \gamma \varepsilon
$$

in a normalised coordinate system, where $\nabla_{\eta}^{\prime}:=\nabla-\eta(\eta \cdot \nabla), \nabla^{\prime}:=\nabla_{e_{n}}^{\prime}$, and $\gamma<1$ is a constant. Repeating the argument for the rescaled solutions, $u_{s^{k}}$, we show that there exists a unit vector $\eta_{0} \in \mathbb{R}^{n}$, such that

$$
\frac{\left\|\nabla_{\eta_{0}}^{\prime} u_{s^{k}}\right\|_{W^{2,2}\left(B_{2}\right)}}{\left\|D^{3} u_{s^{k}}\right\|_{L^{2}\left(B_{1}\right)}} \leq \gamma^{k} \varepsilon
$$


for some $0<s<\gamma<1$. Then the $C^{1, \alpha}$-regularity of the free boundary in a neighbourhood of the origin follows via a standard iteration argument.

From the $C^{1, \alpha}$-regularity of the free boundary it follows that $\Delta u \in C^{1, \alpha}$ up to the free boundary, furthermore, $u$ is $C^{3, \alpha}$ up to the free boundary. Thus a solution $u \in \mathscr{B}_{\kappa}^{\varrho}(\varepsilon)$ is locally $C^{2,1}$, which is the best possible regularity. We provide a two-dimensional counterexample to the $C^{2,1}$-regularity, showing that without our flatness assumptions there exists a solution that is $C^{2, \frac{1}{2}}$ but is not $C^{2, \alpha}$ for $\alpha>\frac{1}{2}$. Hence $C^{2, \frac{1}{2}}$ is the best regularity that a solution may achieve in dimension $n \geq 2$.

\section{The obstacle problem for the biharmonic operator}

This section can be viewed as a background text, where we present the known regularity theory for the biharmonic obstacle problem, with adaptations to our problem. The material in this section is known for the biharmonic obstacle problem with a general obstacle, and zero boundary conditions. Later on in Sect. 3 we will need quantitive estimates for $\|u\|_{W_{l o c}^{3,2}}$ and for $\|u\|_{C_{l o c}^{1, \alpha}}$, hence we decided to include these estimates in the manuscript, with references to the original papers.

\subsection{The known regularity theory for the solution}

The existence and uniqueness of the minimiser of functional (1.1) over the admissible set $\mathscr{A}$ follows by standard arguments in the calculus of variations, see for instance [5]. We give a summary of the known regularity theory for the solution. The following result is due to Frehse, see [1].

Theorem 2.1 (J. Frehse) Let $u$ be the solution to the biharmonic obstacle problem in $\Omega$, where $\Omega \subset \mathbb{R}^{n}$ is an open bounded set. Then

$$
\|u\|_{W^{3,2}(V)} \leq C\|u\|_{W^{2,2}(\Omega)},
$$

for any given $V \subset \subset \Omega$, where $C>0$ is a universal constant, depending only on the space dimension and sets $V, \Omega$, but not on the solution $u$.

Now let us discuss further properties of the solution, referring to the paper by Caffarelli and Friedman [3].

Lemma 2.2 The solution to the biharmonic obstacle problem satisfies the following equation in the distribution sense

$$
\Delta^{2} u=\mu_{u}
$$

where $\mu_{u}$ is a positive measure on $\Omega$.

Proof For any nonnegative test function $\eta \in C_{0}^{\infty}(\Omega)$, the function $u+\varepsilon \eta$ is obviously admissible for any $\varepsilon>0$. Hence $J[u+\varepsilon \eta] \geq J[u]$, consequently

$$
\int \varepsilon^{2}(\Delta \eta)^{2}+2 \varepsilon \Delta u \Delta \eta \geq 0,
$$

and after dividing by $\varepsilon$ and letting $\varepsilon$ go to zero, we obtain

$$
\int \Delta u \Delta \eta \geq 0
$$


for all $\eta \in C_{0}^{\infty}(\Omega), \eta \geq 0$, so $\Delta^{2} u \geq 0$ in the sense of distributions.

Let us study the following linear functional $\Lambda(\eta):=\int_{\Omega} \Delta u \Delta \eta, \eta \in C_{0}^{\infty}(\Omega)$. By the Riesz theorem, $\Lambda$ is a positive measure, let us denote it by $\mu:=\mu_{u}$. Then $\Delta^{2} u=\mu_{u}$ in the sense that

$$
\int_{\Omega} \Delta u \Delta \eta=\int_{\Omega} \eta d \mu_{u}
$$

for every $\eta \in C_{0}^{\infty}(\Omega)$.

Corollary 2.3 There exists an upper semicontinuous function $\omega$ in $\Omega$, such that $\omega=$ $\Delta$ u a.e. in $\Omega$.

Proof For any fixed $x_{0} \in \Omega$, the function

$$
\omega_{r}\left(x_{0}\right):=f_{B_{r}\left(x_{0}\right)} \Delta u(x) d x
$$

is decreasing in $r>0$, since $\Delta u$ is subharmonic by Lemma 2.2. Define $\omega(x):=$ $\lim _{r \rightarrow 0} \omega_{r}(x)$, then $\omega$ is an upper semicontinuous function. On the other hand $\omega_{r}(x) \rightarrow$ $\Delta u(x)$ as $r \rightarrow 0$ a.e., hence $\omega=\Delta u$ a.e. in $\Omega$.

In [2] Frehse proved that $\Delta u \in L_{l o c}^{\infty}$, later on L. Caffarelli and A. Friedman gave another proof to the same result, see Theorem 3.1 in [3]. Below we follow the proof in the latter paper in order to obtain a quantitative estimate on $\|\Delta u\|_{L^{\infty}}$ in our setting. The next lemma is a restatement of the corresponding result in [3], Theorem 2.2.

Lemma 2.4 Let $\Omega \subset \mathbb{R}^{n}$ be a bounded open set with a smooth boundary, and let $u$ be a solution to the biharmonic obstacle problem with zero obstacle. Denote by $S$ the support of the measure $\mu_{u}=\Delta^{2} u$ in $\Omega$, then

$$
\omega\left(x_{0}\right) \geq 0, \text { for every } x_{0} \in S .
$$

Proof The detailed proof of Lemma 2.4 can be found in the original paper [3] and in the book [6, pp. 92-94], so we will provide only a sketch, showing the main ideas.

Extend $u$ to a function in $W_{l o c}^{2,2}\left(\mathbb{R}^{n}\right)$, and denote by $u_{\varepsilon}$ the $\varepsilon$-mollifier of $u$. Let $x_{0} \in \Omega$, assume that there exists a ball $B_{r}\left(x_{0}\right)$, such that $u_{\varepsilon} \geq \alpha>0$ in $B_{r}\left(x_{0}\right)$. Let $\eta \in C_{0}^{\infty}\left(B_{r}\left(x_{0}\right)\right)$, $\eta \geq 0$ and $\eta=1$ in $B_{r / 2}\left(x_{0}\right)$. Then for any $\zeta \in C_{0}^{\infty}\left(B_{r / 2}\left(x_{0}\right)\right)$ and $0<t<\frac{\alpha}{2\|\zeta\|_{\infty}}$ the function

$$
v=\eta u_{\varepsilon}+(1-\eta) u \pm t \zeta
$$

is nonnegative and it satisfies the same boundary conditions as $u$. Hence

$$
\int(\Delta u)^{2} \leq \int\left(\Delta\left(\eta u_{\varepsilon}+(1-\eta) u \pm t \zeta\right)\right)^{2},
$$

after passing to the limit in the last inequality as $\varepsilon \rightarrow 0$, we obtain

$$
\int(\Delta u)^{2} \leq \int(\Delta u \pm t \Delta \zeta)^{2}
$$

Therefore

$$
\int \Delta u \Delta \zeta=0
$$


for all $\zeta \in C_{0}^{\infty}\left(B_{r / 2}\left(x_{0}\right)\right)$, hence $\Delta^{2} u=0$ in $B_{r / 2}\left(x_{0}\right)$ and $x_{0} \notin S$. It follows that if $x_{0} \in S$, then there exists $x_{m} \in \Omega, x_{m} \rightarrow x_{0}$, and $\varepsilon_{m} \rightarrow 0$, such that

$$
u_{\varepsilon_{m}}\left(x_{m}\right) \rightarrow 0, \text { as } m \rightarrow \infty
$$

Then by Green's formula,

$$
u_{\varepsilon_{m}}\left(x_{m}\right)=f_{\partial B_{\rho}\left(x_{m}\right)} u_{\varepsilon_{m}} d \mathcal{H}^{n-1}-\int_{B_{\rho}\left(x_{m}\right)} \Delta u_{\varepsilon_{m}}(y) V\left(x_{m}-y\right) d y,
$$

where $\rho<\operatorname{dist}\left(x_{0}, \partial \Omega\right)$ and $-V(z)$ is Green's function for Laplacian in the ball $B_{\rho}(0)$. Hence

$$
\liminf _{m \rightarrow \infty} \int_{B_{\rho}\left(x_{m}\right)} \Delta u_{\varepsilon_{m}}(y) V\left(x_{m}-y\right) d y \geq 0,
$$

Then it follows from the convergence of the mollifiers and the upper semicontinuity of $\omega$, that $\omega\left(x_{0}\right) \geq 0$, for any $x_{0} \in S$.

Knowing that $\Delta u$ is a subharmonic function, and $\omega \geq 0$ on the support of $\Delta^{2} u$, we can show that $\Delta u$ is locally bounded (Theorem 3.1 in [3]).

Theorem 2.5 Let $u$ be the solution to the biharmonic obstacle problem with zero obstacle in $\Omega, B_{1} \subset \subset \Omega$. Then

$$
\|\Delta u\|_{L^{\infty}\left(B_{1 / 3}\right)} \leq C\|u\|_{W^{2,2}(\Omega)},
$$

where the constant $C>0$ depends on the space dimension $n$ and on $\operatorname{dist}\left(B_{1}, \partial \Omega\right)$.

Proof The detailed proof of the theorem can be found in the original paper [3], Theorem 3.1, and in the book [6, pp. 94-97]. Here we will only provide a sketch of the proof.

Let $\omega$ be the upper semicontinuous equivalent of $\Delta u$ and $x_{0} \in B_{1 / 2}$, then

$$
\omega\left(x_{0}\right) \leq f_{B_{1 / 2}\left(x_{0}\right)} \Delta u(x) d x,
$$

since $\omega$ is a subharmonic function. Applying Hölder's inequality, we obtain

$$
\omega\left(x_{0}\right) \leq\left|B_{1 / 2}\right|^{-\frac{1}{2}}\|\Delta u\|_{L^{2}\left(B_{1}\right)} .
$$

It remains to show that $\Delta u$ is bounded from below in $B_{1 / 2}$. Let $\zeta \in C_{0}^{\infty}\left(B_{1}\right), \zeta=1$ in $B_{2 / 3}$ and $0 \leq \zeta \leq 1$ elsewhere. Referring to [6, p. 96], the following formula holds for any $x \in B_{1 / 2}$

$$
\omega(x)=-\int_{B_{1 / 2}} V(x-y) d \mu-\int_{B_{1} \backslash B_{1 / 2}} \zeta(y) V(x-y) \Delta^{2} u d y+\delta(x),
$$

where $V$ is Green's function for the unit ball $B_{1}$, and $\delta$ is a bounded function,

$$
\|\delta\|_{L^{\infty}\left(B_{1 / 2}\right)} \leq C_{n}\|\Delta u\|_{L^{2}\left(B_{1}\right)} .
$$

Denote

$$
\tilde{V}(x):=\int_{B_{1 / 2}} V(x-y) d \mu(y),
$$


then $\tilde{V}$ is a superharmonic function in $\mathbb{R}^{n}$, and the measure $v:=\Delta \tilde{V}$ is supported on $S_{0}:=B_{1 / 2} \cap S$, moreover according to Lemma 2.4, (2.2)

$$
\tilde{V}(x) \leq-\omega(x)+\delta(x) \leq \delta(x) \text { on } S_{0} .
$$

Taking into account that $\tilde{V}(+\infty)<\infty$, the authors in [3] apply Evans maximum principle, [7] to the superharmonic function $\tilde{V}-\tilde{V}(+\infty)$, and conclude that

$$
\tilde{V}(x) \leq\|\delta\|_{L^{\infty}\left(B_{1 / 2}\right)} \text { in } \mathbb{R}^{n} .
$$

It follows from equation (2.5) that

$$
\omega(x) \geq-\|\delta\|_{L^{\infty}\left(B_{1 / 2}\right)}-c_{n} \mu_{u}\left(B_{1}\right)+\delta(x),
$$

for any $x \in B_{1 / 3}$.

Let $\eta \in C_{0}^{\infty}(\Omega)$ be a nonnegative function, such that $\eta=1$ in $B_{1}$ and $0 \leq \eta \leq 1$ in $\Omega$. Then

$$
\mu_{u}\left(B_{1}\right) \leq \int_{\Omega} \eta d \mu_{u}=\int_{\Omega} \Delta u \Delta \eta \leq\|\Delta u\|_{L^{2}(\Omega)}\|\Delta \eta\|_{L^{2}(\Omega)},
$$

and $\eta$ can be chosen such that $\|\Delta \eta\|_{L^{2}(\Omega)} \leq C\left(\operatorname{dist}\left(B_{1}, \partial \Omega\right)\right)$. Hence

$$
\mu_{u}\left(B_{1}\right) \leq C\|\Delta u\|_{L^{2}(\Omega)},
$$

where the constant $C>0$ depends on the space dimension and on $\operatorname{dist}\left(B_{1}, \partial \Omega\right)$.

Combining the inequalities (2.4) and (2.8) together with (2.9), (2.6), we obtain (2.3).

Corollary 2.6 Let $u$ be the solution to the biharmonic obstacle problem in $\Omega$. Then $u \in C_{l o c}^{1, \alpha}$, for any $0<\alpha<1$, and

$$
\|u\|_{C^{1, \alpha}(K)} \leq C\|u\|_{W^{2,2}(\Omega)},
$$

where the constant $C$ depends on the space dimension and $\operatorname{dist}(K, \partial \Omega)$.

Proof It follows from Theorem 2.5 via a standard covering argument, that

$$
\|\Delta u\|_{L^{\infty}(K)} \leq C\|u\|_{W^{2,2}(\Omega)} .
$$

Then inequality (2.10) follows from the Calderón-Zygmund inequality and the Sobolev embedding theorem.

According to Corollary 2.6, $u$ is a continuous function in $\Omega$, and therefore the noncoincidence set $\Omega_{u}:=\{u>0\}$ is an open subset of $\Omega$. Define the free boundary by

$$
\Gamma_{u}=\partial \Omega_{u} \cap \Omega .
$$

By standard arguments in the theory of free boundary problems, we can see that $u$ is a biharmonic function in the noncoincidence set $\Omega_{u}$. It follows from our discussion that $\mu_{u}=$ $\Delta^{2} u$ is a positive measure supported on $\Gamma_{u}$, and the variational inequalities (1.2) holds with $\varphi \equiv 0$.

\section{Regularity of the free boundary}

In this section we investigate the regularity of the free boundary $\Gamma_{u}$, under the assumption that the solution to the biharmonic obstacle problem is close to the one-dimensional solution $\frac{1}{6}\left(x_{n}\right)_{+}^{3}$. 


\subsection{One-dimensional solutions}

First we find the explicit solution to the biharmonic obstacle problem in the interval $(0,1) \subset$ $\mathbb{R}$.

Example 3.1 The minimiser $u_{0}$ of the functional

$$
J[u]=\int_{0}^{1}\left(u^{\prime \prime}(x)\right)^{2} d x,
$$

over nonnegative functions $u \in W^{2,2}(0,1)$, with boundary conditions $u(0)=1, u^{\prime}(0)=$ $\lambda<-3$ and $u(1)=0, u^{\prime}(1)=0$, is a piecewise 3 -rd order polynomial,

$$
u_{0}(x)=\frac{\lambda^{3}}{3^{3}}\left(x+\frac{3}{\lambda}\right)_{-}^{3}, \quad x \in(0,1),
$$

hence $u_{0} \in C^{2,1}(0,1)$.

Proof Let $u_{0}$ be the minimiser to the given biharmonic obstacle problem. If $0<x_{0}<1$, and $u_{0}\left(x_{0}\right)>0$, then $\int u_{0}^{\prime \prime} \eta^{\prime \prime}=0$, for all infinitely differentiable functions $\eta$ compactly supported in a small ball centered at $x_{0}$. Hence the minimiser $u_{0}$ has a fourth order derivative, $u_{0}^{(4)}(x)=0$ if $x \in\left\{u_{0}>0\right\}$. Therefore $u_{0}$ is a piecewise polynomial of degree less than or equal to three. Denote by $\gamma \in(0,1]$ the first point where the graph of $u_{0}$ hits the $x$-axes. Our aim is find the explicit value of $\gamma$. Then we can also compute the minimiser $u_{0}$.

Observe that $u_{0}(\gamma)=0$, and $u_{0}^{\prime}(\gamma)=0$, since $u_{0}^{\prime}$ is an absolutely continuous function in $(0,1)$. Taking into account the boundary conditions at the points 0 and $\gamma$, we can write $u_{0}(x)=a x^{3}+b x^{2}+\lambda x+1$ in $(0, \gamma)$, where

$$
a=\frac{\lambda \gamma+2}{\gamma^{3}}, b=-\frac{2 \lambda \gamma+3}{\gamma^{2}} .
$$

We see that the point $\gamma$ is a zero of second order for the third order polynomial $u_{0}$, and $u_{0} \geq 0$ in $(0, \gamma]$. That means the third zero is not on the open interval $(0, \gamma)$, hence $\gamma \leq-\frac{3}{\lambda}$.

Consider the function

$$
F(\gamma):=\int_{0}^{\gamma}\left(u^{\prime \prime}(x)\right)^{2} d x
$$

then $F(\gamma)=\frac{4}{\gamma^{3}}\left(\lambda^{2} \gamma^{2}+3 \lambda \gamma+3\right)$. Hence $F^{\prime}(\gamma)=-\frac{4}{\gamma^{4}}(\lambda \gamma+3)^{2}$, showing that the function $F$ is decreasing, so it achieves minimum at the point $\gamma=-\frac{3}{\lambda}$. Therefore we may conclude that

$$
u_{0}(x)=\frac{\lambda^{3}}{3^{3}}\left(x+\frac{3}{\lambda}\right)_{-}^{3}, \quad x \in(0,1)
$$

and $\gamma=-\frac{3}{\lambda}$ is a free boundary point. Observe that $u^{\prime \prime}(\gamma)=0$, and $u^{\prime \prime}$ is a continuous function, but $u^{\prime \prime \prime}$ has a jump discontinuity at the free boundary point $\gamma=-\frac{3}{\lambda}$.

The example above characterises one-dimensional solutions. It also tells us that onedimensional solutions are $C^{2,1}$, and in general are not $C^{3}$. 


\subsection{The class $\mathscr{B}_{K}^{\varrho}(\varepsilon)$ of solutions to the biharmonic obstacle problem}

Without loss of generality, we assume that $0 \in \Gamma_{u}$, and study the regularity of the free boundary, when $u \approx \frac{1}{6}\left(x_{n}\right)_{+}^{3}$.

Let us start by recalling the definition of non-tangentially accessible domains [8].

Definition 3.2 A bounded domain $D \subset \mathbb{R}^{n}$ is called non-tangentially accessible (abbreviated NTA) when there exist constants $M, r_{0}$ and a function $l: \mathbb{R}_{+} \mapsto \mathbb{N}$ such that

1. $D$ satisfies the corkscrew condition; that is for any $x_{0} \in \partial D$ and any $r<r_{0}$, there exists $P=P\left(r, x_{0}\right) \in D$ such that

$$
M^{-1} r<\left|P-x_{0}\right|<r \text { and } \operatorname{dist}(P, \partial D)>M^{-1} r .
$$

2. $D^{c}:=\mathbb{R}^{n} \backslash D$ satisfies the corkscrew condition.

3. Harnack chain condition; if $\epsilon>0$ and $P_{1}, P_{2} \in D$, $\operatorname{dist}\left(P_{i}, \partial D\right)>\epsilon$, and $\left|P_{1}-P_{2}\right|<$ $C \epsilon$, then there exists a Harnack chain from $P_{1}$ to $P_{2}$ whose length $l$ depends on $C$, but not on $\epsilon, l=l(C)$. A Harnack chain from $P_{1}$ to $P_{2}$ is a chain of balls $B_{r_{k}}\left(x^{k}\right), k=1, \ldots, l$ such that $P_{1} \in B_{r_{1}}\left(x^{1}\right), P_{2} \in B_{r_{l}}\left(x^{l}\right), B_{r_{k}}\left(x^{k}\right) \cap B_{r_{k+1}}\left(x^{k+1}\right) \neq \emptyset$, and

$$
M r_{k}>\operatorname{dist}\left(B_{r_{k}}\left(x^{k}\right), \partial D\right)>M^{-1} r_{k} .
$$

Let us define rigorously, what we mean by $u \approx \frac{1}{6}\left(x_{n}\right)_{+}^{3}$.

Definition 3.3 Let $u \geq 0$ be the solution to the biharmonic obstacle problem in a domain $\Omega$, $B_{2} \subset \subset \Omega$ and assume that $0 \in \Gamma_{u}$ is a free boundary point. We say that $u \in \mathscr{B}_{\kappa}^{\varrho}(\varepsilon)$, if the following assumptions are satisfied:

1. $u$ is almost one dimensional, that is

$$
\left\|\nabla^{\prime} u\right\|_{W^{2,2}\left(B_{2}\right)} \leq \varepsilon
$$

where $\nabla^{\prime}:=\nabla-e_{n} \frac{\partial}{\partial x_{n}}$.

2. The set $\Omega_{u}:=\{u>0\}$ is an NTA domain with constants $r_{0}=M^{-1}=\varrho$, and with a function $l$, indicating the length of a Harnack chain.

3. There exists $2>t>0$, such that $u=0$ in $B_{2} \cap\left\{x_{n}<-t\right\}$.

4. We have the following normalisation

$$
\left\|D^{3} u\right\|_{L^{2}\left(B_{1}\right)}=\frac{1}{6}\left\|D^{3}\left(x_{n}\right)_{+}^{3}\right\|_{L^{2}\left(B_{1}\right)}=\frac{\left|B_{1}\right|^{\frac{1}{2}}}{2^{\frac{1}{2}}}:=\omega_{n},
$$

and we also assume that

$$
\left\|D^{3} u\right\|_{L^{2}\left(B_{2}\right)}<\kappa,
$$

where $\kappa>\frac{1}{6}\left\|D^{3}\left(x_{n}\right)_{+}^{3}\right\|_{L^{2}\left(B_{2}\right)}=2^{\frac{n}{2}} \omega_{n}$.

In the notation of the class $\mathscr{B}_{\kappa}^{\varrho}(\varepsilon)$ we did not include the length function $l$, since later it does not appear in our estimates. For the rest of this paper we will assume that we have a fixed length function $l$. Later on in Corollary 3.6 we will see that the precise value of the parameter $t$ in assumption 3 is not very important, and therefore we also omit the parameter $t$ in our notation. From now on $\kappa>2^{\frac{n}{2}} \omega_{n}$ and $1>\varrho>0$ are fixed parameters.

Evidently $\frac{1}{6}\left(x_{n}\right)_{+}^{3} \in \mathscr{B}_{\kappa}^{\varrho}(\varepsilon)$, for any $\varepsilon>0$ and $\varrho>0$. We show that if $u \in \mathscr{B}_{\kappa}^{\varrho}(\varepsilon)$, with $\varepsilon>0$ small, then $u \approx \frac{1}{6}\left(x_{n}\right)_{+}^{3}$ in $W^{3,2}\left(B_{1}\right)$. First we need to prove the following easy lemma. 
Lemma 3.4 Let $u$ be the solution to the biharmonic obstacle problem in $\Omega$. Take $K \subset \subset \Omega$, and a function $\zeta \in C_{0}^{\infty}(K), \zeta \geq 0$, then

$$
\int_{\Omega} \Delta u_{x_{i}} \Delta\left(\zeta u_{x_{i}}\right) \leq 0
$$

for all $i=1,2, \ldots, n$.

Proof Fix $1 \leq i \leq n$, denote $u_{i, h}(x):=u\left(x+h e_{i}\right)$, where $0<|h|<\operatorname{dist}(K, \partial \Omega)$, hence $u_{i, h}$ is defined in $K$. Let us observe that the function $u+t \zeta\left(u_{i, h}-u\right)$ is well defined and nonnegative in $\Omega$ for any $0<t<\frac{1}{\|\zeta\|_{L^{\infty}}}$, and it satisfies the same boundary conditions as $u$. Therefore

$$
\int_{\Omega}\left(\Delta\left(u+t \zeta\left(u_{i, h}-u\right)\right)\right)^{2} \geq \int_{\Omega}(\Delta u)^{2}
$$

after dividing the last inequality by $t$, and taking the limit as $t \rightarrow 0$, we obtain

$$
\int_{K} \Delta u \Delta\left(\zeta\left(u_{i, h}-u\right)\right) \geq 0 .
$$

Note that $u_{i, h}$ is the solution to the biharmonic obstacle problem in $K$, and $u_{i, h}+t \zeta\left(u-u_{i, h}\right)$ is an admissible function, hence

$$
\int_{K}\left(\Delta\left(u_{i, h}+t \zeta\left(u-u_{i, h}\right)\right)\right)^{2} \geq \int_{K}\left(\Delta u_{i, h}\right)^{2},
$$

after dividing the last inequality by $t$, and taking the limit as $t \rightarrow 0$, we obtain

$$
\int_{K} \Delta u_{i, h} \Delta\left(\zeta\left(u-u_{i, h}\right)\right) \geq 0 .
$$

Inequalities (3.9) and (3.10) imply that

$$
\int_{K}\left(\Delta u_{i, h}-\Delta u\right) \Delta\left(\zeta\left(u_{i, h}-u\right)\right) \leq 0,
$$

dividing the last inequality by $h^{2}$, and taking into account that $u \in W_{l o c}^{3,2}$, we may pass to the limit as $|h| \rightarrow 0$ in (3.11), and conclude that

$$
\int_{K} \Delta u_{x_{i}} \Delta\left(\zeta u_{x_{i}}\right) \leq 0
$$

Lemma 3.5 There exists a modulus of continuity $\sigma=\sigma(\varepsilon) \geq 0$, such that

$$
\left\|u(x)-\frac{1}{6}\left(x_{n}\right)_{+}^{3}\right\|_{W^{3,2}\left(B_{1}\right)} \leq \sigma(\varepsilon),
$$

for any $u \in \mathscr{B}_{\kappa}^{\varrho}(\varepsilon)$.

Proof We argue by contradiction. Assume that there exists $\sigma_{0}>0$ and a sequence of solutions, $u^{j} \in \mathscr{B}_{\kappa}^{\varrho}\left(\varepsilon_{j}\right)$, such that

$$
\left\|\nabla^{\prime} u^{j}\right\|_{W^{2,2}\left(B_{2}\right)}=\varepsilon_{j} \rightarrow 0
$$


but

$$
\left\|u^{j}(x)-\frac{1}{6}\left(x_{n}\right)_{+}^{3}\right\|_{W^{3,2}\left(B_{1}\right)}>\sigma_{0}>0 .
$$

According to assumption 4 in Definition 3.3, $\left\|D^{3} u^{j}\right\|_{L^{2}\left(B_{2}\right)}<\kappa$ and according to Assumption 2 the functions $u^{j}$ are vanishing on an open subset of $B_{2}$. Therefore it follows from the Poincaré inequality that $\left\|u^{j}\right\|_{W^{3,2}\left(B_{2}\right)} \leq C(\varrho, n) \kappa$. Hence up to a subsequence $u^{j} \rightarrow u^{0}$ weakly in $W^{3,2}\left(B_{2}\right), u^{j} \rightarrow u^{0}$ strongly in $W^{2,2}\left(B_{2}\right)$ and according to Corollary $2.6 u^{j} \rightarrow u^{0}$ in $C^{1, \alpha}\left(B_{3 / 2}\right)$. Hence

$$
\left\|\nabla^{\prime} u^{0}\right\|_{W^{1,2\left(B_{2}\right)}}=\lim _{j \rightarrow \infty}\left\|\nabla^{\prime} u^{j}\right\|_{W^{1,2}\left(B_{2}\right)} \leq \lim _{j \rightarrow \infty} \varepsilon_{j}=0 .
$$

This implies that $u^{0}$ is a one-dimensional solution (depending only on the variable $x_{n}$ ). Example 3.1 tells us that one-dimensional solutions in the interval $(-2,2)$ have the form

$$
u^{0}\left(x_{n}\right)=c_{1}\left(x_{n}-a_{1}\right)_{-}^{3}+c_{2}\left(x_{n}-a_{2}\right)_{+}^{3},
$$

where $c_{1}, c_{2} \geq 0$ and $-2 \leq a_{1} \leq a_{2} \leq 2$ are constants. According to assumption 3 in Definition 3.3, $u^{0}=c\left(x_{n}-a\right)_{+}^{3}$. In order to obtain a contradiction to Assumption (3.13), we need to show that $u^{j} \rightarrow u_{0}=\frac{1}{6}\left(x_{n}\right)_{+}^{3}$ in $W^{3,2}\left(B_{1}\right)$. The proof of the last statement can be done in two steps.

Step 1 We show that

$$
u^{j} \rightarrow c\left(x_{n}-a\right)_{+}^{3} \text { in } W^{3,2}\left(B_{1}\right) .
$$

Denote $u_{n}^{j}:=\frac{\partial u^{j}}{\partial x_{n}} \in W^{2,2}\left(B_{2}\right), j \in \mathbb{N}_{0}$, and let $\zeta \in C_{0}^{\infty}\left(B_{\frac{3}{2}}\right)$ be a nonnegative function, such that $\zeta \equiv 1$ in $B_{1}$. According to Lemma 3.4,

$$
0 \geq \int_{B_{2}} \Delta\left(\zeta u_{n}^{j}\right) \Delta u_{n}^{j}=\int_{B_{2}} u_{n}^{j} \Delta \zeta \Delta u_{n}^{j}+\int_{B_{2}} \zeta\left(\Delta u_{n}^{j}\right)^{2}+2 \int_{B_{2}} \nabla \zeta \nabla u_{n}^{j} \Delta u_{n}^{j},
$$

and therefore

$$
\begin{aligned}
\limsup _{j \rightarrow \infty} \int_{B_{2}} \zeta\left(\Delta u_{n}^{j}\right)^{2} & \leq-\lim _{j \rightarrow \infty} \int_{B_{2}} u_{n}^{j} \Delta \zeta \Delta u_{n}^{j}-2 \lim _{j \rightarrow \infty} \int_{B_{2}} \nabla \zeta \nabla u_{n}^{j} \Delta u_{n}^{j} \\
& =-\int_{B_{2}} u_{n}^{0} \Delta \zeta \Delta u_{n}^{0}-2 \int_{B_{2}} \nabla \zeta \nabla u_{n}^{0} \Delta u_{n}^{0}=\int_{B_{2}} \zeta\left(\Delta u_{n}^{0}\right)^{2},
\end{aligned}
$$

where in the last step we used integration by parts.

On the other hand, since $\Delta u_{n}^{j} \rightarrow \Delta u_{n}^{0}$ weakly in $L^{2}\left(B_{2}\right)$, it follows that

$$
\liminf _{j \rightarrow \infty} \int_{B_{2}} \zeta\left(\Delta u_{n}^{j}\right)^{2} \geq \int_{B_{2}} \zeta\left(\Delta u_{n}^{0}\right)^{2} .
$$

Therefore, we may conclude from (3.15) and (3.16) that

$$
\lim _{j \rightarrow \infty} \int_{B_{2}} \zeta\left(\Delta u_{n}^{j}\right)^{2}=\int_{B_{2}} \zeta\left(\Delta u_{n}^{0}\right)^{2} .
$$

Hence we obtain

$$
\frac{\partial \Delta u^{j}}{\partial x_{n}} \rightarrow \frac{\partial \Delta u^{0}}{\partial x_{n}} \text { in } L^{2}\left(B_{1}\right) .
$$


Similarly $\frac{\partial \Delta u^{j}}{\partial x_{i}} \rightarrow 0$ in $L^{2}\left(B_{1}\right)$, for $i=1, \ldots, n-1$. Knowing that

$$
\left\|\nabla \Delta u^{j}-\nabla \Delta u^{0}\right\|_{L^{2}\left(B_{1}\right)} \rightarrow 0, \text { and }\left\|u^{j}-u^{0}\right\|_{W^{2,2}\left(B_{2}\right)} \rightarrow 0,
$$

we may apply the Calderón-Zygmund inequality, and conclude (3.14). Recalling that $\left\|D^{3} u^{j}\right\|_{L^{2}\left(B_{1}\right)}=\omega_{n}$, we see that

$$
\left\|D^{3} u^{0}\right\|_{L^{2}\left(B_{1}\right)}=\omega_{n} .
$$

Since $u^{0}=c\left(x_{n}-a\right)_{+}^{3} \geq 0$, it follows that

$$
\left\|D^{3} u^{0}\right\|_{L^{2}\left(B_{1}\right)}^{2}=c^{2} \mathcal{L}^{n}\left(B_{1} \cap\left\{x_{n}>a\right\}\right)>0,
$$

hence

$$
c>0 \text { and } a<1 .
$$

Step 2 We show that $a=0$ and $c=\frac{1}{6}$. Taking into account that $u^{j} \rightarrow u^{0}$ in $C^{1, \alpha}$ and $u^{j}(0)=0$, we conclude that $u^{0}(0)=0$, thus $a \geq 0$. Assume that $a>0$. Since $0 \in \Gamma_{j}$, and $\Omega_{j}$ is an NTA domain, there exists $P_{j}=P(r, 0) \in \Omega_{j}$, for $0<r<\min (\varrho, a / 2)$ as in the corkscrew condition,

$$
\varrho r<\left|P_{j}\right|<r \text { and } \operatorname{dist}\left(P_{j}, \partial \Omega_{j}\right)>\varrho r .
$$

Therefore up to a subsequence $P_{j} \rightarrow P_{0}$, hence $r \varrho \leq\left|P_{0}\right| \leq r, B_{r^{\prime}}\left(P_{0}\right) \subset \Omega_{j}$, for all $j$ large enough, where $0<r^{\prime}<r \varrho$ is a fixed number. Since we have chosen $r<a / 2$, we may conclude that

$$
B_{r^{\prime}}\left(P_{0}\right) \subset\left\{x_{n}<a\right\} \cap \Omega_{j} .
$$

Thus $\Delta u^{j}$ is a sequence of harmonic functions in the ball $B_{r^{\prime}}\left(P_{0}\right)$, and therefore

$$
\Delta u^{j} \rightarrow 0 \text { locally uniformly in } B_{r^{\prime}}\left(P_{0}\right),
$$

according to (3.14).

Let $Q:=e_{n}$, then $u^{0}(Q)=c(1-a)^{3}>0$, since $u^{j} \rightarrow u^{0}$ uniformly in $B_{3 / 2}$, we see that $u^{j}(Q)>0$ for large $j$, and $Q \in \Omega_{j}$. Therefore there exists a Harnack chain connecting $P_{0}$ with $Q ;\left\{B_{r_{1}}\left(x^{1}\right), B_{r_{2}}\left(x^{2}\right), \ldots, B_{r_{l}}\left(x^{l}\right)\right\} \subset \Omega_{j}$, whose length $l$ does not depend on $j$. Denote by $K^{j}:=\cup_{i} B_{r_{i}}\left(x^{i}\right) \subset \subset \Omega_{j}$, and let $V^{j} \subset \subset K^{j} \subset \subset \Omega_{j}$ where $V^{j}$ is a regular domain, such that $\operatorname{dist}\left(K^{j}, \partial V^{j}\right)$ and $\operatorname{dist}\left(V^{j}, \partial \Omega_{j}\right)$ depend only on $r$ and $\varrho$.

Let $w_{+}^{j}$ be a harmonic function in $V^{j}$, with boundary conditions $w_{+}^{j}=\left(\Delta u^{j}\right)_{+} \geq 0$ on $\partial V^{j}$, then $w_{+}^{j}-\Delta u^{j}$ is a harmonic function in $V^{j}$, and $w_{+}^{j}-\Delta u^{j}=\left(\Delta u^{j}\right)_{-} \geq 0$ on $\partial V^{j}$ , hence

$$
0 \leq w_{+}^{j}-\Delta u^{j} \leq\left\|\left(\Delta u^{j}\right)_{-}\right\|_{L^{\infty}\left(V^{j}\right)} \text { in } V^{j} .
$$

Let us observe that $\Delta u^{j} \rightarrow \Delta u^{0}=6 c\left(x_{n}-a\right)_{+}$implies that $\left\|\left(\Delta u^{j}\right)_{-}\right\|_{L^{2}\left(B_{2}\right)} \rightarrow 0$. Since $\left(\Delta u^{j}\right)_{-}$is a subharmonic function in $\Omega_{j}$, and $V^{j} \subset \subset \Omega_{j}$ it follows that

$$
\left\|\left(\Delta u^{j}\right)_{-}\right\|_{L^{\infty}\left(V^{j}\right)} \leq C(n, r, \varrho)\left\|\left(\Delta u^{j}\right)_{-}\right\|_{L^{2}\left(B_{2}\right)} \rightarrow 0 .
$$

So $w_{+}^{j}$ is a nonnegative harmonic function in $V^{j}$, and by the Harnack inequality

$$
C_{H} \inf _{B_{r_{l}}\left(x^{l}\right)} w_{+}^{j} \geq \sup _{B_{r_{l}}\left(x^{l}\right)} w_{+}^{j} \geq w_{+}^{j}\left(e_{n}\right) \geq \Delta u^{j}\left(e_{n}\right) \geq \frac{1}{2} \Delta u_{0}\left(e_{n}\right)=3 c(1-a),
$$


if $j$ is large, where $C_{H}$ is the constant in Harnack's inequality, it depends on $\varrho$ and $r$ but not on $j$. Denote $C(a, c):=3 c(1-a)>0$ by (3.18). Applying the Harnack inequality again, we see that

$$
C_{H} \inf _{B_{r_{l-1}}\left(x^{l-1}\right)} w_{+}^{j} \geq \sup _{B_{r_{l-1}}\left(x^{l-1}\right)} w_{+}^{j} \geq \inf _{B_{r_{l}}\left(x^{l}\right)} w_{+}^{j}>\frac{C(a, c)}{C_{H}} .
$$

Inductively, we obtain that

$$
C_{H} \inf _{B_{r_{1}}\left(x^{1}\right)} w_{+}^{j} \geq \sup _{B_{r_{1}}\left(x^{1}\right)} w_{+}^{j}>\frac{C(a, c)}{C_{H}^{l-1}},
$$

where $l$ does not depend on $j$. Hence $w_{+}^{j}\left(P_{0}\right) \geq \frac{C(a, c)}{C_{H}^{l}}$ for all $j$ large, and according to (3.20),

$$
\lim _{j \rightarrow \infty} \Delta u^{j}\left(P_{0}\right) \geq \frac{C(a, c)}{C_{H}^{l}}>0,
$$

the latter contradicts (3.19). Therefore we may conclude that $a=0$.

Recalling that $\left\|D^{3} u^{0}\right\|_{L^{2}\left(B_{1}\right)}=\omega_{n}$, we see that $c=\frac{1}{6}$, but then we obtain $u^{j} \rightarrow \frac{1}{6}\left(x_{n}\right)_{+}^{3}$ in $W^{3,2}\left(B_{1}\right)$ which is a contradiction, since we assumed (3.13).

Lemma 3.5 has an important corollary, which will be very useful in our later discussion.

Corollary 3.6 Let $u$ be the solution to the biharmonic obstacle problem, $u \in \mathscr{B}_{\kappa}^{\varrho}(\varepsilon)$. Then for any fixed $t>0$ we have that $u(x)=0$ in $B_{2} \cap\left\{x_{n}<-t\right\}$, provided $\varepsilon=\varepsilon(t)>0$ is small.

Proof Once again we argue by contradiction. Assume that there exist $t_{0}>0$ and a sequence of solutions $u_{j} \in \mathscr{B}_{\kappa}^{\varrho}\left(\varepsilon_{j}\right), \varepsilon_{j} \rightarrow 0$, such that $x^{j} \in B_{2} \cap \Gamma_{j}$, and $x_{n}^{j}<-t_{0}$. For $0<r<$ $\min \left(\varrho, t_{0} / 2\right)$ choose $P^{j}=P\left(r, x^{j}\right) \in \Omega_{j}$ as in the corkscrew condition,

$$
r \varrho<\left|x^{j}-P^{j}\right|<r, \quad B_{r \varrho}\left(P^{j}\right) \subset \Omega_{j} .
$$

Upon passing to a subsequence, we may assume that $P^{j} \rightarrow P^{0}$. Fix $0<r^{\prime}<r \varrho$, then for large $j$

$$
B_{r^{\prime}}\left(P^{0}\right) \subset \subset \Omega_{j} \cap\left\{x_{n}<0\right\} .
$$

Hence $\Delta u^{j}$ is a sequence of harmonic functions in $B_{r^{\prime}}\left(P^{0}\right)$. According to Lemma 3.5, $u^{j} \rightarrow \frac{1}{6}\left(x_{n}\right)_{+}^{3}$, and therefore $\Delta u^{j} \rightarrow 0$ in $B_{r^{\prime}}\left(P^{0}\right)$, and $\Delta u^{j}\left(e_{n}\right) \rightarrow 1$. Since $\Omega_{j}$ is an NTA domain, there exists a Harnack chain connecting $P^{0}$ with $Q:=e_{n} \in \Omega_{j}$; $\left\{B_{r_{1}}\left(x^{1}\right), B_{r_{2}}\left(x^{2}\right), \ldots, B_{r_{k}}\left(x^{k}\right)\right\} \subset \Omega_{j}$, whose length does not depend on $j$. Arguing as in the proof of Lemma 3.5, we will obtain a contradiction to $\Delta u_{j} \rightarrow 0$ in $B_{r^{\prime}}\left(P^{0}\right)$.

\subsection{Linearisation}

Let $\left\{u^{j}\right\}$ be a sequence of solutions in $\Omega \supset \supset B_{2}, u^{j} \in \mathscr{B}_{\kappa}^{\varrho}\left(\varepsilon_{j}\right)$, and assume that $\varepsilon_{j} \rightarrow 0$ as $j \rightarrow \infty$. It follows from Lemma 3.5, that up to a subsequence

$$
u^{j} \rightarrow \frac{1}{6}\left(x_{n}\right)_{+}^{3} \text { in } W^{2,2}\left(B_{2}\right) \cap C_{l o c}^{1, \alpha}\left(B_{2}\right) .
$$


Let us denote

$$
\delta_{i}^{j}:=\left\|\frac{\partial u^{j}}{\partial x_{i}}\right\|_{W^{2,2}\left(B_{2}\right)}
$$

Without loss of generality we may assume that $\delta_{i}^{j}>0$, for all $j \in \mathbb{N}$. Indeed, if $\delta_{i}^{j}=0$ for all $j \geq J_{0}$ large, then $u^{j}$ does not depend on the variable $x_{i}$, and the problem reduces to a lower dimensional case. Otherwise we may pass to a subsequence satisfying $\delta_{i}^{j}>0$ for all $j$.

Denote

$$
v_{i}^{j}:=\frac{1}{\delta_{i}^{j}} \frac{\partial u^{j}}{\partial x_{i}}, \quad \text { for } i=1, \ldots, n-1,
$$

then $\left\|v_{i}^{j}\right\|_{W^{2,2}\left(B_{2}\right)}=1$. Therefore up to a subsequence $v_{i}^{j}$ converges to a function $v_{i}^{0}$ weakly in $W^{2,2}\left(B_{2}\right)$ and strongly in $W^{1,2}\left(B_{2}\right)$. For the further discussion we need strong convergence $v_{i}^{j} \rightarrow v_{i}^{0}$ in $W^{2,2}$, at least locally.

Lemma 3.7 Assume that $\left\{u^{j}\right\}$ is a sequence of solutions in $\Omega \supset \supset B_{2}, u^{j} \in \mathscr{B}_{\kappa}^{\varrho}\left(\varepsilon_{j}\right), \varepsilon_{j} \rightarrow 0$. Let $v_{i}^{j}$ be the sequence given by (3.23), and assume that $v_{i}^{j} \rightarrow v_{i}^{0}$ weakly in $W^{2,2}\left(B_{2}\right)$, strongly in $W^{1,2}\left(B_{2}\right)$, for $i=1, \ldots, n-1$, then

$$
\Delta^{2} v_{i}^{0}=0 \text { in } B_{2}^{+}, \quad v_{i}^{0} \equiv 0 \text { in } B_{2} \backslash B_{2}^{+} .
$$

Furthermore, for any $0<R<2$

$$
\left\|v_{i}^{j}-v_{i}^{0}\right\|_{W^{2,2}\left(B_{R}\right)} \rightarrow 0 .
$$

Proof Denote by $\Omega_{j}:=\Omega_{u^{j}}, \Gamma_{j}:=\Gamma_{u^{j}}$. It follows from Corollary 3.6 that $v_{i}^{0} \equiv 0$ in $B_{2} \backslash B_{2}^{+}$, hence $v_{i}^{0}=\left|\nabla v_{i}^{0}\right|=0$ on $\left\{x_{n}=0\right\} \cap B_{2}$ in the trace sense. Moreover, if $K \subset \subset B_{2}^{+}$ is an open subset, then $K \subset \Omega_{j}$ for large $j$ by (3.22). Hence $\Delta^{2} v_{i}^{j}=0$ in $K$, and therefore $\Delta^{2} v_{i}^{0}=0$ in $B_{2}^{+}$, and (3.24) is proved.

Now let us proceed to the proof of the strong convergence. Let $\zeta \in C_{0}^{\infty}\left(B_{2}\right)$ be a nonnegative function, such that $\zeta \equiv 1$ in $B_{R}$ and $0 \leq \zeta \leq 1$ in $B_{2}$. It follows from (3.24) that

$$
0=\int_{B_{2}} \Delta v_{i}^{0} \Delta\left(\zeta v_{i}^{0}\right)=\int_{B_{2}} v_{i}^{0} \Delta \zeta \Delta v_{i}^{0}+\int_{B_{2}} \zeta\left(\Delta v_{i}^{0}\right)^{2}+2 \int_{B_{2}} \nabla \zeta \nabla v_{i}^{0} \Delta v_{i}^{0} .
$$

According to Lemma 3.4

$$
0 \geq \int_{B_{2}} \Delta\left(\zeta v_{i}^{j}\right) \Delta v_{i}^{j}=\int_{B_{2}} v_{i}^{j} \Delta \zeta \Delta v_{i}^{j}+\int_{B_{2}} \zeta\left(\Delta v_{i}^{j}\right)^{2}+2 \int_{B_{2}} \nabla \zeta \nabla v_{i}^{j} \Delta v_{i}^{j},
$$

and therefore

$$
\begin{aligned}
\limsup _{j \rightarrow \infty} \int_{B_{2}} \zeta\left(\Delta v_{i}^{j}\right)^{2} & \leq-\lim _{j \rightarrow \infty} \int_{B_{2}} v_{i}^{j} \Delta \zeta \Delta v_{i}^{j}-2 \lim _{j \rightarrow \infty} \int_{B_{2}} \nabla \zeta \nabla v_{i}^{j} \Delta v_{i}^{j} \\
& =-\int_{B_{2}} v_{i}^{0} \Delta \zeta \Delta v_{i}^{0}-2 \int_{B_{2}} \nabla \zeta \nabla v_{i}^{0} \Delta v_{i}^{0},
\end{aligned}
$$

where we used that $v_{i}^{j} \rightarrow v_{i}^{0}$ in $W^{1,2}\left(B_{2}\right)$ and $\Delta v_{i}^{j} \rightarrow \Delta v_{i}^{0}$ in $L^{2}\left(B_{2}\right)$. 
From the last inequality and (3.26) we may conclude that

$$
\limsup _{j \rightarrow \infty} \int_{B_{2}} \zeta\left(\Delta v_{i}^{j}\right)^{2} \leq \int_{B_{2}} \zeta\left(\Delta v_{i}^{0}\right)^{2} .
$$

On the other hand

$$
\liminf _{j \rightarrow \infty} \int_{B_{2}} \zeta\left(\Delta v_{i}^{j}\right)^{2} \geq \int_{B_{2}} \zeta\left(\Delta v_{i}^{0}\right)^{2}
$$

follows from the weak convergence $\Delta v_{i}^{j} \rightarrow \Delta v_{i}^{0}$ in $L^{2}\left(B_{2}\right)$, and we may conclude from (3.28) and (3.29) that

$$
\lim _{j \rightarrow \infty} \int_{B_{2}} \zeta\left(\Delta v_{i}^{j}\right)^{2}=\int_{B_{2}} \zeta\left(\Delta v_{i}^{0}\right)^{2}
$$

Hence we obtain $\left\|\Delta v_{i}^{j}-\Delta v_{i}^{0}\right\|_{L^{2}\left(B_{R}\right)} \rightarrow 0$, and therefore $v_{i}^{j} \rightarrow v_{i}^{0}$ in $W_{l o c}^{2,2}\left(B_{2}\right)$ according to the Calderón-Zygmund inequality.

\subsection{Properties of solutions in a normalised coordinate system}

Let us define

$$
u_{r, x_{0}}(x):=\frac{u\left(r x+x_{0}\right)}{r^{3}}, \text { for } x_{0} \in \Gamma_{u}, x \in B_{2}, r \in(0,1),
$$

and $u_{r}:=u_{r, 0}$. We would like to know how fast $\left\|\nabla^{\prime} u_{r}\right\|_{W^{2,2}\left(B_{2}\right)}$ decays with respect to $\left\|\nabla^{\prime} u\right\|_{W^{2,2}\left(B_{2}\right)}$, for $r<1$. In particular, the inequality

$$
\left\|\nabla^{\prime} u_{S}\right\|_{W^{2,2}\left(B_{2}\right)} \leq \tau\left\|\nabla^{\prime} u\right\|_{W^{2,2}\left(B_{2}\right)},
$$

for some $0<s, \tau<1$ would provide good decay estimates for $\left\|\nabla^{\prime} u_{s^{k}}\right\|_{W^{2,2}\left(B_{2}\right)}, k \in \mathbb{N}$.

We show that the inequality (3.31) holds in a special coordinate system depending on the solution $u$ and parameter $s>0$. Then iterating the inequality (3.31) and the coordinate system we obtain the existence of the unit normal vector to the free boundary at the origin. $C_{n}$.

Let us observe that $\frac{1}{6}(\eta \cdot x)_{+}^{3} \in \mathscr{B}_{\kappa}^{\varrho}(\varepsilon)$ if $\left|\eta-e_{n}\right| \leq C_{n} \varepsilon$, for some dimensional constant

Definition 3.8 Let $u$ be the solution to the biharmonic obstacle problem. We say that the coordinate system is normalised with respect to $u$, if

$$
\begin{gathered}
\inf _{\eta \in \mathbb{R}^{n},|\eta|=1}\left\|\nabla_{\eta}^{\prime}\left(u(x)-\frac{1}{6}(\eta \cdot x)_{+}^{3}\right)\right\|_{L^{2}\left(B_{2}\right)} \\
=\left\|\nabla_{e_{n}}^{\prime}\left(u(x)-\frac{1}{6}\left(x_{n}\right)_{+}^{3}\right)\right\|_{L^{2}\left(B_{2}\right)},
\end{gathered}
$$

where $\nabla_{\eta}^{\prime}:=\nabla-(\eta \cdot \nabla) \eta$, and $\nabla^{\prime}:=\nabla_{e_{n}}^{\prime}$.

A minimiser $\eta$ always exists for a function $u \in \mathscr{B}_{\kappa}^{\varrho}(\varepsilon)$, and since $\nabla_{-\eta}^{\prime}=\nabla_{\eta}^{\prime},-\eta$ is also a minimiser, thus we always choose a minimiser satisfying the condition $e_{n} \cdot \eta \geq 0$. A normalised coordinate system always exists by choosing $\eta=e_{n}$ in the new coordinate system. 
Lemma 3.9 Let $u$ be the solution to the biharmonic obstacle problem in a normalised coordinate system with respect to $u$. Then

$$
\int_{B_{2}} \frac{\partial u}{\partial x_{i}} \frac{\partial u}{\partial x_{n}} d x=0, \quad \text { for all } 1 \leq i \leq n-1 .
$$

Proof Let us observe that for every $\eta \in \mathbb{R}^{n}$,

$$
\nabla_{\eta}^{\prime}\left(u(x)-\frac{1}{6}(\eta \cdot x)_{+}^{3}\right)=\nabla_{\eta}^{\prime} u(x)
$$

and

$$
\left\|\nabla_{\eta}^{\prime} u\right\|_{L^{2}\left(B_{2}\right)}^{2}=\|\nabla u\|_{L^{2}\left(B_{2}\right)}^{2}-\|\eta \cdot \nabla u\|_{L^{2}\left(B_{2}\right)}^{2} .
$$

For any fixed $1 \leq i \leq n-1$, and real number $-1<t<1$, let $\eta(t):=t e_{i}+\sqrt{1-t^{2}} e_{n}$. By the definition of a normalised coordinate system, the function $\varphi(t):=\|\eta \cdot \nabla u\|_{L^{2}\left(B_{2}\right)}^{2}$, $t \in(-1,1)$ has a local maximum at the point $t=0$. Hence

$$
\varphi^{\prime}(0)=2 \int_{B_{2}} \frac{\partial u}{\partial x_{i}} \frac{\partial u}{\partial x_{n}} d x=0,
$$

which implies (3.32).

Lemma 3.10 Assume that $u \in \mathscr{B}_{\kappa}^{\varrho}(\varepsilon)$ solves the biharmonic obstacle problem in a fixed coordinate system with basis vectors $\left\{e_{1}, \ldots, e_{n}\right\}$. Let $\left\{e_{1}^{1}, \ldots, e_{n}^{1}\right\}$ be a normalised coordinate system with respect to $u$, and assume that $e_{n}^{1} \cdot e_{n} \geq 0$. Then

$$
\left|e_{n}-e_{n}^{1}\right| \leq C(n)\left\|\nabla^{\prime} u\right\|_{L^{2}\left(B_{2}\right)} \leq C(n) \varepsilon,
$$

if $\varepsilon$ is small, where $C(n)>0$ is a dimensional constant.

Proof According to Definition 3.8,

$$
\left\|\nabla_{e_{n}^{1}}^{\prime} u\right\|_{L^{2}\left(B_{2}\right)}=\left\|\nabla u-e_{n}^{1}\left(e_{n}^{1} \cdot \nabla u\right)\right\|_{L^{2}\left(B_{2}\right)} \leq\left\|\nabla^{\prime} u\right\|_{L^{2}\left(B_{2}\right)} .
$$

It follows from the triangle inequality that

$$
\begin{aligned}
& \left\|\frac{\partial u}{\partial x_{n}}-\left(e_{n} \cdot e_{n}^{1}\right)^{2} \frac{\partial u}{\partial x_{n}}\right\|_{L^{2}\left(B_{2}\right)} \leq\left\|\frac{\partial u}{\partial x_{n}}-\left(e_{n} \cdot e_{n}^{1}\right)\left(e_{n}^{1} \cdot \nabla u\right)\right\|_{L^{2}\left(B_{2}\right)} \\
& \quad+\left\|\left(e_{n} \cdot e_{n}^{1}\right)\left(e_{n}^{1} \cdot \nabla u\right)-\left(e_{n} \cdot e_{n}^{1}\right)^{2} \frac{\partial u}{\partial x_{n}}\right\|_{L^{2}\left(B_{2}\right)} \\
& \leq\left\|\nabla_{e_{n}^{1}}^{\prime} u\right\|_{L^{2}\left(B_{2}\right)}+\left(e_{n} \cdot e_{n}^{1}\right)\left\|e_{n}^{1} \cdot \nabla^{\prime} u\right\|_{L^{2}\left(B_{2}\right)} \leq 2\left\|\nabla^{\prime} u\right\|_{L^{2}\left(B_{2}\right)},
\end{aligned}
$$

according to (3.34), and taking into account that $0 \leq e_{n} \cdot e_{n}^{1} \leq 1$.

Note that Lemma 3.5 implies that $\left\|\frac{\partial u}{\partial x_{n}}\right\|_{L^{2}\left(B_{2}\right)} \approx\left\|\frac{x_{n}^{2}}{2}\right\|_{L^{2}\left(B_{2}^{+}\right)}$is uniformly bounded from below by a dimensional constant if $\varepsilon>0$ is small. We may conclude from (3.35) that

$$
1-\left(e_{n} \cdot e_{n}^{1}\right)^{2} \leq \frac{2\left\|\nabla^{\prime} u\right\|_{L^{2}\left(B_{2}\right)}}{\left\|\frac{\partial u}{\partial x_{n}}\right\|_{L^{2}\left(B_{2}\right)}} \leq C(n)\left\|\nabla^{\prime} u\right\|_{L^{2}\left(B_{2}\right)} .
$$

Since $0 \leq e_{n} \cdot e_{n}^{1} \leq 1$, we get

$$
0 \leq 1-e_{n} \cdot e_{n}^{1} \leq 1-\left(e_{n} \cdot e_{n}^{1}\right)^{2} \leq C(n)\left\|\nabla^{\prime} u\right\|_{L^{2}\left(B_{2}\right)} .
$$


Denote by $\left(e_{n}^{1}\right)^{\prime}:=e_{n}^{1}-e_{n}\left(e_{n} \cdot e_{n}^{1}\right)$. It follows from the triangle inequality and (3.34) that

$$
\begin{aligned}
& \left\|\left(e_{n}^{1}\right)^{\prime}\left(e_{n}^{1} \cdot \nabla u\right)\right\|_{L^{2}\left(B_{2}\right)} \leq\left\|\nabla^{\prime} u-\left(e_{n}^{1}\right)^{\prime}\left(e_{n}^{1} \cdot \nabla u\right)\right\|_{L^{2}\left(B_{2}\right)} \\
& \quad+\left\|\nabla^{\prime} u\right\|_{L^{2}\left(B_{2}\right)} \leq\left\|\nabla_{e_{n}^{1}}^{\prime} u\right\|_{L^{2}\left(B_{2}\right)}+\left\|\nabla^{\prime} u\right\|_{L^{2}\left(B_{2}\right)} \leq 2\left\|\nabla^{\prime} u\right\|_{L^{2}\left(B_{2}\right)} .
\end{aligned}
$$

Hence

$$
\left|\left(e_{n}^{1}\right)^{\prime}\right| \leq \frac{2\left\|\nabla^{\prime} u\right\|_{L^{2}\left(B_{2}\right)}}{\left\|e_{n}^{1} \cdot \nabla u\right\|_{L^{2}\left(B_{2}\right)}} .
$$

Let us choose $\varepsilon>0$ small, then $\left\|e_{n}^{1} \cdot \nabla u\right\|_{L^{2}\left(B_{2}\right)}$ is bounded from below by a dimensional constant according to Lemma 3.5 and inequality (3.36). Therefore we obtain

$$
\left|\left(e_{n}^{1}\right)^{\prime}\right| \leq C(n)\left\|\nabla^{\prime} u\right\|_{L^{2}\left(B_{2}\right)} .
$$

Note that

$$
\left|e_{n}-e_{n}^{1}\right| \leq\left|1-e_{n} \cdot e_{n}^{1}\right|+\left|\left(e_{n}^{1}\right)^{\prime}\right| .
$$

Applying inequalities (3.36) and (3.37) we obtain the desired inequality,

$$
\left|e_{n}-e_{n}^{1}\right| \leq C(n)\left\|\nabla^{\prime} u\right\|_{L^{2}\left(B_{2}\right)} \leq C(n) \varepsilon,
$$

and the proof of the lemma is now complete.

Lemma 3.10 provides an essential estimate, which will be useful in our later discussion. Next we state another supporting lemma, the proof of which is quite standard, but we include it for our convenience.

Lemma 3.11 1. Let $v$ be a biharmonic function in the ball $B_{2}$, then

$$
\|\Delta v\|_{L^{2}\left(B_{1}\right)} \leq C_{n}\|v\|_{L^{2}\left(B_{2}\right)} .
$$

2. If $v$ is a biharmonic function in the half-ball $B_{2}^{+}$, such that $v=|\nabla v|=0$ on $\left\{x_{n}=0\right\} \cap B_{2}$, then

$$
\|\Delta v\|_{L^{2}\left(B_{1}^{+}\right)} \leq C_{n}\|v\|_{L^{2}\left(B_{2}^{+}\right)} .
$$

Proof Throughout $\zeta \in C_{0}^{\infty}\left(B_{2}\right)$ is a fixed function, such that $\zeta \equiv 1$ in $B_{1}, 0 \leq \zeta \leq 1$ in $B_{2}$.

1. If $v$ is a biharmonic function in $B_{2}$, then

$$
\begin{aligned}
0= & \int_{B_{2}} \Delta v \Delta\left(\zeta^{4} v\right)=\int_{B_{2}} 2 v\left(\Delta \zeta^{2}+4|\nabla \zeta|^{2}\right) \zeta^{2} \Delta v \\
& +\int_{B_{2}} \zeta^{4}(\Delta v)^{2}+8 \int_{B_{2}} \zeta \nabla \zeta \nabla v \zeta^{2} \Delta v .
\end{aligned}
$$

Hence by Cauchy's inequality,

$$
\begin{aligned}
\int_{B_{2}} \zeta^{4}(\Delta v)^{2}= & -\int_{B_{2}} 2 v\left(\Delta \zeta^{2}+4|\nabla \zeta|^{2}\right) \zeta^{2} \Delta v-8 \int_{B_{2}} \zeta \nabla \zeta \nabla v \zeta^{2} \Delta v \\
\leq & \frac{1}{4} \int_{B_{2}} \zeta^{4}(\Delta v)^{2}+4 \int_{B_{2}} v^{2}\left(\Delta \zeta^{2}+4|\nabla \zeta|^{2}\right)^{2}+\frac{1}{4} \int_{B_{2}} \zeta^{4}(\Delta v)^{2} \\
& +64 \int_{B_{2}}(\zeta \nabla \zeta \nabla v)^{2} \leq \frac{1}{2} \int_{B_{2}} \zeta^{4}(\Delta v)^{2}+C_{n}\|v\|_{L^{2}\left(B_{2}\right)}^{2}+C_{n}\|\zeta \nabla v\|_{L^{2}\left(B_{2}\right)}^{2}
\end{aligned}
$$


On the other hand,

$$
\begin{aligned}
\int_{B_{2}} \zeta^{2}|\nabla v|^{2}= & \int_{B_{2}} \nabla\left(\zeta^{2} v\right) \nabla v-2 \int_{B_{2}} \zeta v \nabla \zeta \nabla v \\
= & -\int_{B_{2}} \zeta^{2} v \Delta v-2 \int_{B_{2}} \zeta v \nabla \zeta \nabla v \leq \frac{1}{8 C_{n}} \int_{B_{2}} \zeta^{4}(\Delta v)^{2}+2 C_{n} \int_{B_{2}} v^{2} \\
& +\frac{1}{2} \int_{B_{2}} \zeta^{2}|\nabla v|^{2}+2 \int_{B_{2}}|\nabla \zeta|^{2} v^{2}
\end{aligned}
$$

and therefore

$$
C_{n}\|\zeta \nabla v\|_{L^{2}\left(B_{2}\right)}^{2} \leq \frac{1}{4} \int_{B_{2}} \zeta^{4}(\Delta v)^{2}+\tilde{C}_{n} \int_{B_{2}} v^{2}
$$

Combining estimates (3.40) and (3.41), we obtain

$$
\int_{B_{2}} \zeta^{4}(\Delta v)^{2} \leq \frac{3}{4} \int_{B_{2}} \zeta^{4}(\Delta v)^{2}+\bar{C}_{n}\|v\|_{L^{2}\left(B_{2}\right)}^{2},
$$

which implies (3.38).

2. In order to prove the second part of the lemma, it is enough to observe that

$$
\int_{B_{2}^{+}} \Delta v \Delta\left(\zeta^{4} v\right)=0
$$

since $\zeta^{4} v \in W_{0}^{2,2}\left(B_{2}^{+}\right)$. The rest of the proof follows as in the first part.

Proposition 3.12 For any small number $0<s<2^{-n-4} e^{-1} n^{-2}$, there exists $\varepsilon_{0}=\varepsilon_{0}(s)>0$ small, such that if $\varepsilon<\varepsilon_{0}$, then for any $u \in \mathscr{B}_{K}^{\varrho}(\varepsilon)$

$$
\left\|\nabla^{\prime} u_{2 s}\right\|_{L^{2}\left(B_{2}\right)} \leq C_{n}\left\|\nabla^{\prime} u\right\|_{W^{2,2}\left(B_{2}\right)},
$$

where $C_{n}$ is a dimensional constant, not depending on s. Furthermore, if the coordinate system is normalised with respect to $u_{2 s}$, then

$$
\left\|\nabla^{\prime} u_{s}\right\|_{W^{2,2}\left(B_{2}\right)} \leq \tau\left\|\nabla^{\prime} u\right\|_{W^{2,2}\left(B_{2}\right)},
$$

where $1>\tau>\Lambda_{n} s$ is a fixed number and $\Lambda_{n}$ is a dimensional constant to be specified.

Proof The proof of inequalities (3.42) and (3.43) follows the exact same procedure, so we will mainly focus on the proof of the second one, since it is the core of the linearisation argument.

We want to show that the inequality (3.43) holds in a normalised coordinate system with respect to $u_{2 s}$. By the Cauchy-Schwartz inequality, it is enough to show that the inequality

$$
\left\|\frac{\partial u_{s}}{\partial x_{i}}\right\|_{W^{2,2}\left(B_{2}\right)} \leq \tau\left\|\frac{\partial u}{\partial x_{i}}\right\|_{W^{2,2}\left(B_{2}\right)}
$$

holds for any $i \in\{1, \ldots, n-1\}$, provided $\varepsilon$ is small enough. We argue by contradiction. Assume that there exists small numbers $0<s<2^{-n-4} e^{-1} n^{-2}, \Lambda_{n} s<\tau<1$ and a sequence of solutions $\left\{u^{j}\right\} \subset \mathscr{B}_{\kappa}^{\varrho}\left(\varepsilon_{j}\right)$, in a coordinate system normalised with respect to $u_{2 s}^{j}$, such that $\varepsilon_{j} \rightarrow 0$, as $j \rightarrow \infty$, but for some $i \in\{1,2, \ldots, n-1\}$

$$
\left\|\frac{\partial u_{s}^{j}}{\partial x_{i}}\right\|_{W^{2,2}\left(B_{2}\right)}>\tau\left\|\frac{\partial u^{j}}{\partial x_{i}}\right\|_{W^{2,2}\left(B_{2}\right)} .
$$


Let $v_{i}^{j}$ be given by (3.23), then according to Lemma 3.7, $v_{i}^{j} \rightarrow v_{i}^{0}$ in $W_{l o c}^{2,2}\left(B_{2}\right)$, where $v_{i}^{0}$ is a biharmonic function in the half-ball $\left\{x_{n}>0\right\} \cap B_{2}$, satisfying $v_{i}^{0}=\left|\nabla v_{i}^{0}\right|=0$ on $\left\{x_{n}=0\right\} \cap B_{2}$. Inequality (3.44) implies that

$$
\left\|\frac{v_{i}^{0}(s \cdot)}{s^{2}}\right\|_{W^{2,2}\left(B_{2}\right)} \geq \tau \text {. }
$$

Lemma 3.11, part 2. and the Calderón-Zygmund inequality imply that

$$
\left\|\frac{v_{i}^{0}(2 s \cdot)}{4 s^{2}}\right\|_{W^{2,2}\left(B_{1}\right)} \leq C_{n}\left\|\frac{v_{i}^{0}(2 s \cdot)}{4 s^{2}}\right\|_{L^{2}\left(B_{2}\right)},
$$

hence

$$
\left\|\frac{v_{i}^{0}(2 s \cdot)}{4 s^{2}}\right\|_{L^{2}\left(B_{2}\right)} \geq \frac{1}{C_{n}}\left\|\frac{v_{i}^{0}(2 s \cdot)}{4 s^{2}}\right\|_{W^{2,2}\left(B_{1}\right)} \geq C_{n}\left\|\frac{v_{i}^{0}(s \cdot)}{s^{2}}\right\|_{W^{2,2}\left(B_{2}\right)} \geq C_{n} \tau,
$$

where $C_{n}$ represents a general dimensional constant, and it does not depend neither on the function $v_{i}^{0}$ nor on the parameter $s$. We will derive a contradiction to (3.45), if we show that $\left\|\frac{v_{i}^{0}(2 s \cdot)}{4 s^{2}}\right\|_{L^{2}\left(B_{2}\right)}$ can be made arbitrarily small by choosing $s>0$ small initially.

Since $v_{i}^{0}$ is a biharmonic function in the half-ball $\left\{x_{n}>0\right\} \cap B_{2}$ and $v_{i}^{0}=\left|\nabla v_{i}^{0}\right|=0$ on $\left\{x_{n}=0\right\} \cap B_{2}$, we can apply the reflection principle for biharmonic functions, and extend $v_{i}^{0}$ to a biharmonic function in the ball $B_{2}$, see for instance [9] or [10]. Let $\bar{v}_{i}^{0}$ denote the extended function given by Duffin's formula

$$
\bar{v}_{i}^{0}\left(x^{\prime},-x_{n}\right)=-v_{i}^{0}\left(x^{\prime}, x_{n}\right)+2 x_{n} \frac{\partial v_{i}^{0}}{\partial x_{n}}\left(x^{\prime}, x_{n}\right)-x_{n}^{2} \Delta v_{i}^{0}\left(x^{\prime}, x_{n}\right), x_{n}>0 .
$$

The formula (3.47) implies that

$$
\left\|\bar{v}_{i}^{0}\right\|_{L^{2}\left(B_{2}\right)} \leq c_{n}\left\|v_{i}^{0}\right\|_{W^{2,2}\left(B_{2}\right)},
$$

where $c_{n}>0$ is yet another dimensional constant.

The function $\bar{v}_{i}^{0}$ is biharmonic in the ball $B_{2}$, therefore analytic and it may be written as a Taylor series

$$
\bar{v}_{i}^{0}(x)=\sum_{|\alpha|=0}^{\infty} \frac{D^{\alpha} \bar{v}_{i}^{0}(0)}{\alpha !} x^{\alpha}=\sum_{k=0}^{\infty} b_{k}(x),
$$

where $\alpha$ is a multiindex, and $b_{k}$ is a homogeneous degree $k$ biharmonic polynomial. It follows from boundary conditions for the function $v_{i}^{0}$ on $\left\{x_{n}=0\right\}$ that

$$
b_{0}=b_{1} \equiv 0 \text {, and } b_{2}(x)=\frac{\partial^{2} \bar{v}_{i}^{0}(0)}{\partial x_{n}^{2}} \frac{x_{n}^{2}}{2} .
$$

Lemma 3.5 implies that $\frac{\partial u^{j}}{\partial x_{n}} \rightarrow \frac{1}{2}\left(x_{n}^{+}\right)^{2}$ in $L^{2}\left(B_{2}\right)$, and according to Lemma 3.7, $\frac{v_{i}^{j}(2 s x)}{4 s^{2}} \rightarrow \frac{v_{i}^{0}(2 s x)}{4 s^{2}}$ in $W^{2,2}\left(B_{2}\right)$ as $j \rightarrow \infty$, and $v_{i}^{0}=0$ in $B_{2} \backslash B_{2}^{+}$. By Lemma 3.9,

$$
\int_{B_{2}} v_{i}^{j}(2 s x) \frac{\partial u^{j}}{\partial x_{n}}(2 s x) d x=\frac{1}{\delta_{i}^{j}} \int_{B_{2}} \frac{\partial u^{j}}{\partial x_{i}}(2 s x) \frac{\partial u^{j}}{\partial x_{n}}(2 s x) d x=0,
$$


and after passing to the limit as $j \rightarrow \infty$, we obtain that

$$
\int_{B_{2}^{+}} v_{i}^{0}(2 s x) x_{n}^{2} d x=0
$$

Note that (3.51) implies that

$$
\left\|\frac{v_{i}^{0}(2 s \cdot)}{4 s^{2}}-b_{2}\right\|_{L^{2}\left(B_{2}^{+}\right)}^{2}=\left\|\frac{v_{i}^{0}(2 s \cdot)}{4 s^{2}}\right\|_{L^{2}\left(B_{2}^{+}\right)}^{2}+\left\|b_{2}\right\|_{L^{2}\left(B_{2}^{+}\right)}^{2},
$$

hence

$$
\left\|\frac{v_{i}^{0}(2 s \cdot)}{4 s^{2}}\right\|_{L^{2}\left(B_{2}^{+}\right)} \leq\left\|\frac{v_{i}^{0}(2 s \cdot)}{4 s^{2}}-b_{2}\right\|_{L^{2}\left(B_{2}^{+}\right)} .
$$

Next we show that $\left\|v_{i}^{0}(2 s \cdot)-4 s^{2} b_{2}\right\|_{L^{2}\left(B_{2}^{+}\right)}^{2}$ is of order $s^{3}$. By the triangle inequality,

$$
\begin{aligned}
\left\|\frac{v_{i}^{0}(2 s \cdot)}{4 s^{2}}-b_{2}\right\|_{L^{2}\left(B_{2}^{+}\right)} & =\left\|\sum_{k=3}^{\infty}(2 s)^{-2} b_{k}(2 s \cdot)\right\|_{L^{2}\left(B_{2}^{+}\right)} \\
\leq \sum_{k=3}^{\infty}\left\|(2 s)^{-2} b_{k}(2 s \cdot)\right\|_{L^{2}\left(B_{2}^{+}\right)} & =\sum_{k=3}^{\infty}(2 s)^{k-2}\left\|b_{k}\right\|_{L^{2}\left(B_{2}^{+}\right)} .
\end{aligned}
$$

Now it is time to refer to the estimates on derivatives for biharmonic functions (see "Appendix A"),

$$
\begin{aligned}
b_{k}(x) & =\sum_{|\alpha|=k} \frac{D^{\alpha} \bar{v}_{0}^{i}(0)}{\alpha !} x^{\alpha}, \text { and } \\
\left|D^{\alpha} \bar{v}_{0}^{i}(0)\right| \leq & \frac{\left(2^{n+1} n k\right)^{k}}{r^{n+k}}\left(\left\|\bar{v}_{i}^{0}\right\|_{L^{1}\left(B_{r}\right)}+\frac{r^{2}}{2(n+2)}\left\|\Delta \bar{v}_{i}^{0}\right\|_{L^{1}\left(B_{r}\right)}\right) .
\end{aligned}
$$

Hence

$$
\begin{aligned}
\left\|b_{k}\right\|_{L^{2}\left(B_{2}^{+}\right)} & \leq \sum_{|\alpha|=k} \frac{\left(2^{n+1} n k\right)^{k} 2^{n+k}}{\left|B_{1}\right|^{\frac{1}{2}} \alpha !}\left(\left\|\bar{v}_{i}^{0}\right\|_{L^{1}\left(B_{1}\right)}+\frac{1}{2(n+2)}\left\|\Delta \bar{v}_{i}^{0}\right\|_{L^{1}\left(B_{1}\right)}\right) \\
& \stackrel{(3.38)}{\leq} C_{n}\left(2^{n+2} n k\right)^{k} \sum_{|\alpha|=k} \frac{1}{\alpha !}\left\|\bar{v}_{i}^{0}\right\|_{L^{2}\left(B_{2}\right)}=C_{n} \frac{\left(2^{n+2} n k\right)^{k} n^{k}}{k !}\left\|\bar{v}_{i}^{0}\right\|_{L^{2}\left(B_{2}\right)} \\
& \leq C_{n} 2^{k(n+2)} n^{2 k} e^{k}\left\|\bar{v}_{i}^{0}\right\|_{L^{2}\left(B_{2}\right)},
\end{aligned}
$$

where we used Stirling's inequality in the last step.

Let $\lambda:=2^{n+2} e n^{2}$ be a fixed number, then by (3.53),

$$
\begin{aligned}
\left\|\frac{v_{i}^{0}(2 s \cdot)}{4 s^{2}}-b_{2}\right\|_{L^{2}\left(B_{2}^{+}\right)} & \leq C_{n} s^{-2} \sum_{k=3}^{\infty}(2 s)^{k} \lambda^{k}\left\|\bar{v}_{i}^{0}\right\|_{L^{2}\left(B_{2}\right)} \\
& =C_{n} \frac{\lambda^{3} s}{1-2 \lambda s}\left\|\bar{v}_{i}^{0}\right\|_{L^{2}\left(B_{2}\right)} \leq \tilde{C}_{n} s\left\|\bar{v}_{i}^{0}\right\|_{L^{2}\left(B_{2}\right)},
\end{aligned}
$$

where by assumption $2 s \lambda<1 / 2$. 
Finally, combining the inequalities (3.48), (3.52) and (3.55), we obtain

$$
\left\|\frac{v_{i}^{0}(2 s \cdot)}{4 s^{2}}\right\|_{L^{2}\left(B_{2}\right)} \leq c_{n} \tilde{C}_{n} s\left\|v_{i}^{0}\right\|_{W^{2,2}\left(B_{2}\right)} \leq A_{n} s,
$$

where $A_{n}>0$ is a dimensional constant, and $s$ is fixed small number, $s A_{n}<1$. Let $\Lambda_{n}:=A_{n} / C_{n}$, where $C_{n}$ is the dimensional constant in (3.46). Recalling that $1>\tau>s \Lambda_{n}$, we derive a contradiction to (3.46).

The proof of the inequality (3.42) is very similar. Any biharmonic function $v$ in the half ball $B_{2}^{+}$, satisfying the boundary conditions $v=|\nabla v|=0$ on $\left\{x_{n}=0\right\}$ can be written as (3.49). Employing the estimates of derivatives of biharmonic functions, we can show that $\left\|\frac{v(s \cdot)}{s^{2}}\right\|_{L^{2}\left(B_{2}\right)}$ is bounded by a dimensional constant if $0<s<2^{-n-2} e^{-1} n^{-2}$, and (3.42) follows.

\section{$3.5 C^{1, \alpha}$-regularity of the free boundary}

In this section we perform an iteration argument, based on Proposition 3.12 and Lemma 3.10, that leads to the existence of the unit normal $\eta_{0}$ of the free boundary at the origin, and provides good decay estimates for $\left\|\nabla_{\eta_{0}}^{\prime} u_{r}\right\|_{W^{2,2}\left(B_{2}\right)}$.

First we would like to verify that $u \in \mathscr{B}_{K}^{\varrho}(\varepsilon)$ imply that $u_{s} \in \mathscr{B}_{K}^{\varrho}(\varepsilon)$. It is easy to check that the property of being an NTA domain is scaling invariant, in the sense that if $D$ is an NTA domain and $0 \in \partial D$, then for any $0<s<1$ the set $D_{s}:=s^{-1}\left(D \cap B_{s}\right)$ is also an NTA domain with the same parameters as $D$.

Assumption 3 in Definition 3.3 holds for $u_{s}$ according to Corollary 3.6. Indeed, let $t=s$ in Corollary 3.6, then $u(s x)=0$ if $x_{n}<-1$.

Thus $u_{s}$ satisfies 2, 3 in Definition 3.3, but it may not satisfy 4. Instead we consider rescaled solutions defined as follows

$$
U_{s}(x):=\frac{\omega_{n} u_{s}(x)}{\left\|D^{3} u_{s}\right\|_{L^{2}\left(B_{1}\right)}},
$$

then assumption 4 also holds. Indeed, $\left\|D^{3} U_{s}\right\|_{L^{2}\left(B_{1}\right)}=\omega_{n}$ by definition of $U_{s}$, and

$$
\begin{aligned}
\left\|D^{3} U_{s}\right\|_{L^{2}\left(B_{2}\right)} & =\frac{\omega_{n}\left\|D^{3} u_{s}\right\|_{L^{2}\left(B_{2}\right)}}{\left\|D^{3} u_{s}\right\|_{L^{2}\left(B_{1}\right)}}=\frac{\omega_{n}\left\|D^{3} u\right\|_{L^{2}\left(B_{2 s}\right)}}{\left\|D^{3} u\right\|_{L^{2}\left(B_{s}\right)}} \\
& \leq \omega_{n} \frac{\omega_{n}(2 s)^{\frac{n}{2}}+\sigma(\varepsilon)}{\omega_{n}(s)^{\frac{n}{2}}-\sigma(\varepsilon)}<\kappa,
\end{aligned}
$$

according to Lemma 3.5 provided $\varepsilon=\varepsilon(n, \kappa, s)$ is small.

In the next lemma we show that $U_{s} \in \mathscr{B}_{\kappa}^{\varrho}(\gamma \varepsilon)$ in a normalised coordinate system, then we argue inductively to show that $U_{s^{k}} \in \mathscr{B}_{\kappa}^{\varrho}\left(\gamma^{k} \varepsilon\right), \gamma<1$.

Lemma 3.13 Assume that $u \in \mathscr{B}_{\kappa}^{\varrho}(\varepsilon)$ solves the biharmonic obstacle problem in a normalised coordinate system $\left\{e_{1}, e_{2}, \ldots, e_{n}\right\}$. Then for any $0<\alpha<1$ there exist $r_{0}>0$ and a unit vector $\eta_{0} \in \mathbb{R}^{n}$, such that $\left|\eta_{0}-e_{n}\right| \leq C \varepsilon$, and for any $0<r<r_{0}$

$$
\frac{\left\|\nabla_{\eta_{0}}^{\prime} u_{r}\right\|_{W^{2,2}\left(B_{2}\right)}}{\left\|D^{3} u_{r}\right\|_{L^{2}\left(B_{1}\right)}} \leq C r^{\alpha} \varepsilon,
$$

provided $\varepsilon=\varepsilon(n, \kappa, \varrho, \alpha)$ is small enough. The constant $C>0$ depends only on the given parameters. 
Proof Throughout $\left\{e_{1}, \ldots, e_{n}\right\}$ is a fixed coordinate system normalised with respect to the solution $u \in \mathscr{B}_{\kappa}^{\varrho}(\varepsilon)$, and $\nabla^{\prime} u=\nabla_{e_{n}}^{\prime} u$. We may renormalise the coordinate system with respect to $U_{2 s}$ and denote by $\left\{e_{1}^{1}, \ldots, e_{n}^{1}\right\}$ the set of basis vectors in the new system. Inductively, $\left\{e_{1}^{k}, \ldots, e_{n}^{k}\right\}, k \in \mathbb{N}$ is a normalised system with respect to $U_{2 s^{k}}$, and $e_{i}^{0}:=e_{i}$. According to Lemma 3.10,

$$
\left|e_{n}^{k+1}-e_{n}^{k}\right| \leq C(n) \frac{\left\|\nabla_{e_{n}^{k}}^{\prime} u_{2 s^{k+1}}\right\|_{L^{2}\left(B_{2}\right)}}{\left\|D^{3} u_{s^{k}}\right\|_{L^{2}\left(B_{1}\right)}} \stackrel{(3.42)}{\leq} C(n) \frac{\left\|\nabla_{e_{n}^{k}}^{\prime} u_{s^{k}}\right\|_{W^{2,2}\left(B_{2}\right)}}{\left\|D^{3} u_{s^{k}}\right\|_{L^{2}\left(B_{1}\right)}}
$$

provided $\left\|\nabla_{e_{n}^{k}}^{\prime} U_{s^{k}}\right\|_{W^{2,2}\left(B_{2}\right)}$ is sufficiently small.

In the following discussion $0<s<\tau<1$ are small fixed numbers, satisfying the assumptions in Proposition 3.12.

Now let us consider the sequence of numbers $\left\{A_{k}\right\}_{k \in \mathbb{N}_{0}}$, defined as follows:

$$
A_{k}:=\frac{\omega_{n}\left\|\nabla_{e_{n}^{k}}^{\prime} u_{s^{k}}\right\|_{W^{2,2}\left(B_{2}\right)}}{\left\|D^{3} u_{s^{k}}\right\|_{L^{2}\left(B_{1}\right)}}, \text { for } k=0,1,2, \ldots
$$

By definition, $A_{0} \leq \varepsilon$, and

$$
A_{1}=\frac{\omega_{n}\left\|\nabla_{e_{n}^{1}}^{\prime} u_{S}\right\|_{W^{2,2}\left(B_{2}\right)}}{\left\|D^{3} u_{s}\right\|_{L^{2}\left(B_{1}\right)}}=\frac{\left\|D^{3} u\right\|_{L^{2}\left(B_{1}\right)}}{\left\|D^{3} u_{S}\right\|_{L^{2}\left(B_{1}\right)}}\left\|\nabla_{e_{n}^{1}}^{\prime} u_{S}\right\|_{W^{2,2}\left(B_{2}\right)} .
$$

Applying Proposition 3.12 and Lemma 3.10 for a function $u \in \mathscr{B}_{K}^{\varrho}(\varepsilon)$, we obtain

$$
\begin{aligned}
& \left\|\nabla_{e_{n}^{1}}^{\prime} u_{s}\right\|_{W^{2,2}\left(B_{2}\right)} \leq \tau\left\|\nabla_{e_{n}^{1}}^{\prime} u\right\|_{W^{2,2}\left(B_{2}\right)} \\
& \quad \leq \tau\left(\left\|\nabla^{\prime} u\right\|_{W^{2,2}\left(B_{2}\right)}+2\left|e_{n}-e_{n}^{1}\right|\|\nabla u\|_{W^{2,2}\left(B_{2}\right)}\right) \\
& \quad \stackrel{(3.58)}{\leq} \tau\left(\left\|\nabla^{\prime} u\right\|_{W^{2,2}\left(B_{2}\right)}+2 C(n) \frac{\left\|\nabla^{\prime} u\right\|_{W^{2,2}\left(B_{2}\right)}}{\left\|D^{3} u\right\|_{L^{2}\left(B_{1}\right)}}\|\nabla u\|_{W^{2,2}\left(B_{2}\right)}\right) \\
& \quad \stackrel{(3.42)}{\leq} \tau C(n, \kappa)\left\|\nabla^{\prime} u\right\|_{W^{2,2}\left(B_{2}\right)} .
\end{aligned}
$$

Let $\beta:=\tau C(n, \kappa)$, and $\beta<\gamma<1$ be fixed numbers. Then

$$
\begin{aligned}
\frac{\left\|D^{3} u\right\|_{L^{2}\left(B_{1}\right)}}{\left\|D^{3} u_{s}\right\|_{L^{2}\left(B_{1}\right)}} & =\frac{s^{\frac{n}{2}}\left\|D^{3} u\right\|_{L^{2}\left(B_{1}\right)}}{\left\|D^{3} u\right\|_{L^{2}\left(B_{S}\right)}} \\
& \leq \frac{s^{\frac{n}{2}} \omega_{n}}{s^{\frac{n}{2}} \omega_{n}-\sigma\left(A_{0}\right)} \leq \frac{\gamma}{\beta} .
\end{aligned}
$$

according to Lemma 3.5, provided $A_{0} \leq \varepsilon$ is small depending on the parameter $s$ and dimension $n$. The last inequality together with (3.60) and (3.61) implies that $A_{1} \leq \gamma \varepsilon$.

We use an induction argument to show that

$$
A_{k} \leq \gamma^{k} \varepsilon, \quad \text { for all } k \in \mathbb{N}_{0}
$$

for fixed $1>\gamma>\beta>\tau>\Lambda_{n} s>0$. Assuming that (3.62) holds for $k \in \mathbb{N}$, we will show that $A_{k+1} \leq \gamma A_{k}$.

By the induction assumption

$$
\left\|\nabla_{e_{n}^{k}}^{\prime} U_{s^{k}}\right\|_{W^{2,2}\left(B_{2}\right)}=\frac{\omega_{n}\left\|\nabla_{e_{n}^{k}}^{\prime} u_{s^{k}}\right\|_{W^{2,2}\left(B_{2}\right)}}{\left\|D^{3} u_{s^{k}}\right\|_{L^{2}\left(B_{1}\right)}}=A_{k} \leq \gamma^{k} \varepsilon .
$$


Hence

$$
U_{s^{k}}=\frac{\omega_{n} u_{s^{k}}(x)}{\left\|D^{3} u_{s^{k}}\right\|_{L^{2}\left(B_{1}\right)}} \in \mathscr{B}_{\kappa}^{\varrho}\left(\gamma^{k} \varepsilon\right)
$$

in the coordinate system $\left\{e_{1}^{k}, \ldots, e_{n}^{k}\right\}$. By definition, $\left\{e_{1}^{k+1}, \ldots, e_{n}^{k+1}\right\}$ is a normalised coordinate system with respect to $U_{2 s^{k+1}} \in \mathscr{B}_{\kappa}^{\varrho}\left(\beta^{k} \varepsilon\right)$, and by (3.43)

$$
A_{k+1}=\frac{\omega_{n}\left\|\nabla_{e_{n}^{k+1}}^{\prime} u_{s^{k+1}}\right\|_{W^{2,2}\left(B_{2}\right)}}{\left\|D^{3} u_{s^{k+1}}\right\|_{L^{2}\left(B_{1}\right)}} \leq \frac{\omega_{n} \tau\left\|\nabla_{e_{n}^{k+1}}^{\prime} u_{s^{k}}\right\|_{W^{2,2}\left(B_{2}\right)}}{\left\|D^{3} u_{s^{k}}\right\|_{L^{2}\left(B_{1}\right)}} \frac{\left\|D^{3} u_{s^{k}}\right\|_{L^{2}\left(B_{1}\right)}}{\left\|D^{3} u_{s^{k+1}}\right\|_{L^{2}\left(B_{1}\right)}} .
$$

First we observe that

$$
\begin{aligned}
\frac{\left\|D^{3} u_{s^{k}}\right\|_{L^{2}\left(B_{1}\right)}}{\left\|D^{3} u_{s^{k+1}}\right\|_{L^{2}\left(B_{1}\right)}} & =\frac{s^{\frac{n}{2}}\left\|D^{3} u_{s^{k}}\right\|_{L^{2}\left(B_{1}\right)}}{\left\|D^{3} u_{s^{k}}\right\|_{L^{2}\left(B_{s}\right)}} \\
& \leq \frac{s^{\frac{n}{2}} \omega_{n}}{s^{\frac{n}{2}} \omega_{n}-\sigma\left(\gamma^{k} A_{0}\right)} \leq \frac{\gamma}{\beta},
\end{aligned}
$$

according to Lemma 3.5, since $U_{s^{k}} \in \mathscr{B}_{\kappa}^{\varrho}\left(\gamma^{k} \varepsilon\right)$ and $\gamma^{k} \varepsilon<\varepsilon$ is small.

Next we estimate

$$
\begin{aligned}
& \left\|\nabla_{e_{n}^{k+1}}^{\prime} u_{s^{k}}\right\|_{W^{2,2}\left(B_{2}\right)} \leq\left\|\nabla_{e_{n}^{k}}^{\prime} u_{s^{k}}\right\|_{W^{2,2}\left(B_{2}\right)}+2\left|e_{n}^{k+1}-e_{n}^{k}\right|\left\|\nabla u_{s^{k}}\right\|_{W^{2,2}\left(B_{2}\right)} \\
& \quad \stackrel{(3.58)}{\leq}\left\|\nabla_{e_{n}^{k}}^{\prime} u_{s^{k}}\right\|_{W^{2,2}\left(B_{2}\right)}+\frac{C(n)\left\|\nabla_{e_{n}^{k}}^{\prime} u_{s^{k}}\right\|_{W^{2,2}\left(B_{2}\right)}}{\left\|D^{3} u_{s^{k}}\right\|_{L^{2}\left(B_{1}\right)}}\left\|\nabla u_{s^{k}}\right\|_{W^{2,2}\left(B_{2}\right)} \\
& \quad \stackrel{(3.42)}{\leq} C(n, \kappa)\left\|\nabla_{e_{n}^{k}}^{\prime} u_{s^{k}}\right\|_{W^{2,2}\left(B_{2}\right)} .
\end{aligned}
$$

Finally we obtain from (3.65) and (3.66) that

$$
A_{k+1} \leq \frac{\omega_{n} \tau C(n, \kappa) \gamma}{\beta} \frac{\left\|\nabla_{e_{n}^{k}}^{\prime} u_{s^{k}}\right\|_{W^{2,2}\left(B_{2}\right)}}{\left\|D^{3} u_{s^{k}}\right\|_{L^{2}\left(B_{1}\right)}}=\gamma A_{k} \leq \gamma^{k+1} A_{0},
$$

this completes the proof of inequality (3.62).

Next we show that $\left\{e_{n}^{k}\right\}$ is a Cauchy sequence by using (3.58) and (3.62). Indeed for any $m, k \in \mathbb{N}$,

$$
\begin{aligned}
\left|e_{n}^{k+m}-e_{n}^{k}\right| & \leq \sum_{l=1}^{m}\left|e_{n}^{k+l}-e_{n}^{k+l-1}\right| \leq C(n) \sum_{l=1}^{m}\left\|\nabla_{e_{n}^{k+l-1}}^{\prime} U_{2 s^{k+l}}\right\|_{L^{2}\left(B_{2}\right)} \\
& \leq C(n) \sum_{l=1}^{m} A_{k+l-1} \leq C(n) A_{0} \sum_{l=1}^{m} \gamma^{k+l-1} \leq \frac{C(n) A_{0}}{(1-\gamma)} \gamma^{k},
\end{aligned}
$$

hence $e_{n}^{k} \rightarrow \eta_{0}$, as $k \rightarrow \infty$ for some $\eta_{0} \in \mathbb{R}^{n},\left|\eta_{0}\right|=1$ and

$$
\left|\eta_{0}-e_{n}^{k}\right| \leq C^{\prime}(n) A_{0} \gamma^{k} \leq C^{\prime}(n) \gamma^{k} \varepsilon,
$$

in particular $\left|\eta_{0}-e_{n}\right| \leq C^{\prime}(n) \varepsilon$.

Now the inequality (3.57) follows via a standard iteration argument. Let $0<\alpha<1$ be any number, choose $s=s(n, \alpha)$ small, satisfying the assumption in Proposition 3.12, and 
such that $\gamma=C_{n} s<s^{\alpha}$. If $0<r \leq s$, then there exists $k \in \mathbb{N}_{0}$, such that $s^{k+1} \leq r<s^{k}$. Hence

$$
\begin{aligned}
& \frac{\left\|\nabla_{\eta_{0}}^{\prime} u_{r}\right\|_{W^{2,2}\left(B_{2}\right)}}{\left\|D^{3} u_{r}\right\|_{L^{2}\left(B_{1}\right)}} \leq C \frac{\left\|\nabla_{\eta_{0}}^{\prime} u_{s^{k}}\right\|_{W^{2,2}\left(B_{2}\right)}}{\left\|D^{3} u_{s^{k}}\right\|_{L^{2}\left(B_{1}\right)}} \\
& \quad \leq C \frac{\left\|\nabla_{e_{n}^{k}}^{\prime} u_{s^{k}}\right\|_{W^{2,2}\left(B_{2}\right)}+2\left|e_{n}^{k}-\eta_{0}\right|\left\|\nabla u_{s^{k}}\right\|_{W^{2,2}\left(B_{2}\right)}}{\left\|D^{3} u_{s^{k}}\right\|_{L^{2}\left(B_{1}\right)}} \leq C \gamma^{k} \varepsilon \leq C s^{\alpha k} \varepsilon \leq C r^{\alpha} \varepsilon,
\end{aligned}
$$

where $C$ depends on the space dimension and on the given parameters.

Now we are ready to prove the $C^{1, \alpha}$-regularity of the free boundary.

Theorem 3.14 Let $0<\alpha<1$ be a given number. Assume that $u \in \mathscr{B}_{\kappa}^{\varrho}(\varepsilon)$, with an $\varepsilon>0$ small, depending on $\alpha$ and the space dimension. Then there exists $0<r_{0}<1$ depending on the given parameters, such that $\Gamma_{u} \cap B_{r_{0}}$ is a $C^{1, \alpha}$-graph and the $C^{1, \alpha}$-norm of the graph is bounded by $C \varepsilon$.

Proof Let $0<\alpha<1$ and fix $s=s(n, \alpha)>0$ small as in (3.69). It follows from Lemma 3.13 that for $u \in \mathscr{B}_{K}^{\varrho}(\varepsilon)$

$$
\frac{\left\|\nabla^{\prime} u_{r}\right\|_{W^{2,2}\left(B_{2}\right)}}{\left\|D^{3} u_{r}\right\|_{L^{2}\left(B_{1}\right)}} \leq C r^{\alpha} \rightarrow 0 \text { as } r \rightarrow 0
$$

after a change of variable, by choosing $e_{n}=\eta_{0}$, where $\eta_{0}$ is the same vector as in Lemma 3.13. Then

$$
\frac{\omega_{n} u_{r}(x)}{\left\|D^{3} u_{r}\right\|_{L^{2}\left(B_{1}\right)}} \rightarrow \frac{1}{6}\left(x_{n}\right)_{+}^{3}
$$

according to Lemma 3.5 .

So we have shown that in the initial coordinate system,

$$
\frac{\omega_{n} u(r x)}{r^{3}\left\|D^{3} u_{r}\right\|_{L^{2}\left(B_{1}\right)}} \rightarrow \frac{1}{6}\left(\eta_{0} \cdot x\right)_{+}^{3} \text { in } W^{3,2}\left(B_{1}\right) \cap C^{1, \alpha}\left(B_{1}\right), \text { as } r \rightarrow 0,
$$

and therefore $\eta_{0}$ is the measure theoretic normal to $\Gamma_{u}$ at the origin.

Now let $x_{0} \in \Gamma_{u} \cap B_{s}$ be a free boundary point, and consider the function $u_{x_{0}, 1 / 2}(x)=$ $\frac{u\left(x / 2+x_{0}\right)}{(1 / 2)^{3}}, x \in B_{2}$, then

$$
U_{x_{0}}(x):=\frac{\omega_{n} u_{x_{0}, 1 / 2}(x)}{\left\|D^{3} u_{x_{0}, 1 / 2}\right\|_{L^{2}\left(B_{1}\right)}} \in \mathscr{B}_{\kappa}^{\varrho}(C(n) \varepsilon) .
$$

According to Lemma 3.13, $U_{x_{0}}$ has a unique blow-up

$$
U_{r, x_{0}}(x):=\frac{U_{x_{0}}(r x)}{r^{3}}=\frac{\omega_{n} u_{x_{0}, 1 / 2}(r x)}{r^{3}\left\|D^{3} u_{x_{0}, 1 / 2}(r x)\right\|_{L^{2}\left(B_{1}\right)}} \rightarrow \frac{1}{6}\left(\eta_{x_{0}} \cdot x\right)_{+}^{3} .
$$

and therefore $\eta_{x_{0}}$ is the normal to $\Gamma_{u}$ at $x_{0}$.

Next we show that $\eta_{x}$ is a Hölder continuous function on $\Gamma_{u} \cap B_{s}$. If $x_{0} \in \Gamma_{u} \cap B_{s}$, then $s^{k+1}<\left|x_{0}\right| \leq s^{k}$, for some $k \in \mathbb{N}_{0}$. Hence $\left\|\nabla_{\eta_{0}}^{\prime} U_{s^{k}, x_{0}}\right\|_{W^{2,2}\left(B_{2}\right)} \leq C \gamma^{k} \varepsilon$, and $\left\|\nabla_{\eta_{x_{0}}}^{\prime} U_{r, x_{0}}\right\|_{W^{2,2}\left(B_{1}\right)} \rightarrow 0$ as $r \rightarrow 0$. Applying Lemma 3.13 for the function $U_{s^{k}, x_{0}} \in$ $\mathscr{B}_{\kappa}^{\varrho}\left(C \gamma^{k} \varepsilon\right)$, we obtain

$$
\left|\eta_{x_{0}}-\eta_{0}\right| \leq C \gamma^{k} \varepsilon \leq \frac{C}{\gamma}\left|x_{0}\right|^{\alpha} \varepsilon
$$


Furthermore, the inequality

$$
\left|\eta_{x}-\eta_{y}\right| \leq C|x-y|^{\alpha} \varepsilon, \quad \text { for any } x, y \in \Gamma_{u}
$$

follows from (3.71).

\section{On the regularity of the solution}

In this section we study the regularity of the solution to the biharmonic obstacle problem. Assuming that $u \in \mathscr{B}_{\kappa}^{\varrho}(\varepsilon)$, with $\varepsilon>0$ small, we derive from Theorem 3.14 that $u \in C_{l o c}^{2,1}\left(B_{1}\right)$. In the end we provide an example showing that without the NTA domain assumption, there exist solutions, which are not $C^{2,1}$.

\section{1 $C^{2,1}$-regularity of the solutions in $\mathscr{B}_{K}^{\varrho}(\varepsilon)$}

After showing the $C^{1, \alpha}$-regularity of the free boundary $\Gamma_{u} \cap B_{r_{0}}$, we may go further to derive improved regularity for the solution $u \in \mathscr{B}_{\kappa}^{\varrho}(\varepsilon)$.

Theorem 4.1 Let $u \in \mathscr{B}_{\kappa}^{\varrho}(\varepsilon)$ be the solution to the biharmonic obstacle problem in $\Omega \supset \supset B_{2}$, and let $0<\alpha<1$ be a fixed number. Then there exists $r_{0}>0$ such that $u \in C^{2,1}\left(B_{r_{0}}\right)$, provided $\varepsilon=\varepsilon(\kappa, \varrho, \alpha)$ is small. Furthermore, the following estimate holds

$$
\|u\|_{C^{3, \alpha}\left(\bar{\Omega}_{u} \cap B_{r_{0}}\right)} \leq C(n)\|u\|_{W^{2,2}\left(B_{2}\right)} \leq C(n) \kappa,
$$

where $C(n)$ is just a dimensional constant.

Proof According to Theorem 3.14, $\Gamma_{u} \cap B_{s}$ is a graph of a $C^{1, \alpha}$-function. We know that $\Delta u \in W^{1,2}\left(B_{2}\right)$ is a harmonic function in $\Omega_{u}:=\{u>0\}$, and also $u \in W^{3,2}\left(B_{2}\right), u \equiv 0$ in $\Omega \backslash \Omega_{u}$, hence $\Delta u=0$ on $\Gamma_{u}=\partial \Omega_{u} \cap B_{2}$ in the trace sense. Therefore we may apply Corollary 8.36 in [11], to conclude that $\Delta u \in C^{1, \alpha}\left(\left(\Omega_{u} \cup \Gamma_{u}\right) \cap B_{3 s / 4}\right)$, and

$$
\|\Delta u\|_{C^{1, \alpha}\left(\bar{\Omega}_{u} \cap B_{3 s / 4}\right)} \leq C(n)\|\Delta u\|_{L^{\infty}\left(B_{1}\right)} .
$$

It follows from the Calderón-Zygmund estimates that $u \in W^{3, p}\left(B_{s / 2}\right)$, for any $p<\infty$. According to the Sobolev embedding theorem, $u \in C^{2, \alpha}\left(B_{S / 2}\right)$, for all $\alpha<1$, with the following estimate

$$
\|u\|_{C^{2, \alpha}\left(\overline{\Omega_{u}} \cap B_{s / 2}\right)} \leq C(n)\left(\|\Delta u\|_{C^{1, \alpha}\left(\bar{\Omega}_{u} \cap B_{3 s / 4}\right)}+\|u\|_{C^{1, \alpha}\left(B_{3 / 4}\right)}\right) .
$$

Denote by $u_{i j}:=\frac{\partial^{2} u}{\partial x_{i} \partial x_{j}}$. Then $u_{i j} \in W^{1,2}\left(B_{1}\right) \cap C^{\alpha}\left(\Omega_{u} \cap B_{3 s / 4}\right)$ is a weak solution of $\Delta u_{i j}=\frac{\partial f_{j}}{\partial x_{i}}$ in $\Omega_{u} \cap B_{3 s / 4}$, where $f_{j}:=\frac{\partial \Delta u}{\partial x_{j}} \in C^{\alpha}\left(\Omega_{u} \cap B_{3 / 4}\right)$. Taking into account that $u_{i j}=0$ on $\partial \Omega_{u} \cap B_{1 / 2}$, we may apply Corollary 8.36 in [11] once again and conclude that

$$
\left\|u_{i j}\right\|_{C^{1, \alpha}\left(\bar{\Omega}_{u} \cap B_{s / 4}\right)} \leq C(n)\left(\left\|u_{i j}\right\|_{C^{0}\left(\bar{\Omega}_{u} \cap B_{s / 2}\right)}+\|\Delta u\|_{C^{1, \alpha}\left(\bar{\Omega}_{u} \cap B_{3 s / 4}\right)}\right),
$$

hence

$$
\left\|D^{2} u\right\|_{C^{1, \alpha}\left(\bar{\Omega}_{u} \cap B_{s / 4}\right)} \leq C_{n}^{\prime}\left(\|\Delta u\|_{C^{1, \alpha}\left(\bar{\Omega}_{u} \cap B_{3 s / 4}\right)}+\|u\|_{C^{1, \alpha}\left(B_{3 / 4}\right)}\right),
$$

according to (4.2). 
Therefore we obtain

$$
\begin{aligned}
\|u\|_{C^{3, \alpha}\left(\bar{\Omega}_{u} \cap B_{s / 4}\right)} & \leq\|u\|_{C^{1, \alpha}\left(B_{3 / 4}\right)}+\left\|D^{2} u\right\|_{C^{1, \alpha}\left(\bar{\Omega}_{u} \cap B_{s / 4}\right)} \\
& \leq C(n)\left(\|\Delta u\|_{C^{1, \alpha}\left(\bar{\Omega}_{u} \cap B_{3 s / 4}\right)}+\|u\|_{C^{1, \alpha}\left(B_{3 s / 4}\right)}\right) .
\end{aligned}
$$

Taking into account that

$$
\left\|D^{3} u\right\|_{L^{\infty}\left(B_{s / 4}\right)} \leq\left\|D^{3} u\right\|_{C^{0, \alpha}\left(\bar{\Omega}_{u} \cap B_{s / 4}\right)},
$$

we see that $u \in C^{2,1}\left(B_{S / 4}\right)$.

\subsection{In general the solutions are not better than $C^{2, \frac{1}{2}}$}

Let us observe that the assumption $u \in \mathscr{B}_{\kappa}^{\varrho}(\varepsilon)$ is essential in the proof of $u \in C^{2,1}\left(B_{r}\right)$. The next example shows that without our flatness assumptions there exists a solution to the biharmonic obstacle problem in $\mathbb{R}^{2}$, that do not possess $C^{2,1}$ - regularity.

Example 4.2 Consider the following function given in polar coordinates in $\mathbb{R}^{2}$,

$$
u(r, \varphi)=r^{\frac{5}{2}}\left(\cos \frac{\varphi}{2}-\frac{1}{5} \cos \frac{5 \varphi}{2}\right), r \in[0,1), \varphi \in[-\pi, \pi)
$$

then $u \in C^{2, \frac{1}{2}}$ is the solution to the biharmonic zero-obstacle problem in the unit ball $B_{1} \subset \mathbb{R}^{2}$.

Proof It is easy to check that $u \geq 0, u(x)=0$ if and only if $-1 \leq x_{1} \leq 0$ and $x_{2}=0$. Hence the set $\Omega_{u}=\{u>0\}$ is not an NTA domain, since the complement of $\Omega_{u}$ does not satisfy the corkscrew condition.

Let us show that $\Delta^{2} u$ is a nonnegative measure supported on $[-1,0] \times\{0\}$. For any nonnegative $f \in C_{0}^{\infty}\left(B_{1}\right)$, we compute

$$
\begin{aligned}
\int_{B_{1}} \Delta u(x) \Delta f(x) d x & =\int_{0}^{1} \int_{-\pi}^{\pi} r \Delta f(r, \varphi) \Delta u(r, \varphi) d \varphi d r \\
& =6 \int_{0}^{1} \int_{-\pi}^{\pi} r^{\frac{3}{2}} \Delta f(r, \varphi) \cos \frac{\varphi}{2} d \varphi d r=6 \int_{0}^{1} r^{-\frac{1}{2}} f(r, \pi) d r \geq 0,
\end{aligned}
$$

where we used integration by parts, and that $f$ is compactly supported in $B_{1}$.

We obtain that $u$ solves the following variational inequality,

$$
u \geq 0, \Delta^{2} u \geq 0, u \cdot \Delta^{2} u=0 .
$$

Now we show that $u$ is the unique minimiser to the following zero-obstacle problem: minimize the functional (1.1) over

$$
\mathscr{A}:=\left\{v \in W^{2,2}\left(B_{1}\right), v \geq 0 \text {, s.t. } v=u, \frac{\partial v}{\partial n}=\frac{\partial u}{\partial n}, \text { on } \partial B_{1}\right\} \neq \varnothing .
$$

The functional $J$ admits a unique minimiser over $\mathscr{A}$, let us call it $v$. It follows from (4.4), that

$$
\int_{B_{1}} \Delta u \Delta(v-u)=\int_{B_{1}}(v-u) \Delta^{2} u=\int_{B_{1}} v \Delta^{2} u \geq 0 .
$$


Hence

$$
\int_{B_{1}}(\Delta u)^{2} \leq \int_{B_{1}} \Delta u \Delta v \leq\left(\int_{B_{1}}(\Delta u)^{2}\right)^{\frac{1}{2}}\left(\int_{B_{1}}(\Delta v)^{2}\right)^{\frac{1}{2}},
$$

where we used the Hölder inequality in the last step. Therefore we obtain

$$
\int_{B_{1}}(\Delta u)^{2} \leq \int_{B_{1}}(\Delta v)^{2},
$$

thus $u \equiv v$, and $u$ solves the biharmonic zero-obstacle problem in the unit ball.

However $\Delta u=6 r^{\frac{1}{2}} \cos \frac{\varphi}{2}$, which implies that $u$ is $C^{2, \frac{1}{2}}$, and that the exponent $\frac{1}{2}$ is optimal, in particular $u$ is not $C^{2,1}$.

Acknowledgements Open access funding provided by Royal Institute of Technology. First of all I wish to thank my doctoral thesis advisor, John Andersson, for suggesting this research project and for all his help and encouragement. I am grateful to Erik Lindgren for reading preliminary versions of the manuscript and thereby improving it significantly. I would like to thank the referee for pointing out a gap in one of the proofs, which has been corrected in the current version.

Open Access This article is distributed under the terms of the Creative Commons Attribution 4.0 International License (http://creativecommons.org/licenses/by/4.0/), which permits unrestricted use, distribution, and reproduction in any medium, provided you give appropriate credit to the original author(s) and the source, provide a link to the Creative Commons license, and indicate if changes were made.

\section{A Estimates on derivatives of biharmonic functions}

In this part of the paper estimates on derivatives of biharmonic functions are obtained. We believe that these estimates are known, but we could not find a reference, and therefore included in the paper.

Lemma A.1 Let $v$ be a biharmonic function in the ball $B_{1} \subset \mathbb{R}^{n}$, and assume that $B_{r}\left(x_{0}\right) \subset$ $B_{1}$. Then

$$
\left|D^{\alpha} v\left(x_{0}\right)\right| \leq \frac{\left(2^{n+1} n k\right)^{k}}{\left|B_{1}\right| r^{n+k}}\left(\|v\|_{L^{1}\left(B_{r}\left(x_{0}\right)\right)}+\frac{r^{2}}{2(n+2)}\|\Delta v\|_{L^{1}\left(B_{r}\left(x_{0}\right)\right)}\right),
$$

where $\alpha$ is a multiindex, and $k=|\alpha|$.

Proof The following mean value properties are known for biharmonic functions

$$
v\left(x_{0}\right)=f_{\partial B_{r}\left(x_{0}\right)} v d S-\frac{r^{2}}{2 n} \Delta v\left(x_{0}\right)
$$

and

$$
v\left(x_{0}\right)=f_{B_{r}\left(x_{0}\right)} v d x-\frac{r^{2}}{2(n+2)} \Delta v\left(x_{0}\right) .
$$

The proofs of (A.2) and (A.3) are similar to the proofs of the mean value properties for harmonic functions. For a fixed $x_{0}$, let $\phi(r):=f_{\partial B_{r}\left(x_{0}\right)} v d S$. It is easy to see by Green's formula that

$$
\phi^{\prime}(r)=\frac{r}{n} f_{B_{r}\left(x_{0}\right)} \Delta v d x=\frac{r}{n} \Delta v\left(x_{0}\right)
$$


where we also used the mean value property for the harmonic function $\Delta v$. Hence (A.2) follows by integrating (A.4) in the interval $(0, r)$.

Now (A.3) can be shown by using (A.2) and the co-area formula;

$$
\begin{aligned}
f_{B_{r}\left(x_{0}\right)} v d x & =\left|B_{r}\right|^{-1} \int_{0}^{r} \int_{\partial B_{s}\left(x_{0}\right)} v d S d s=\int_{0}^{r} \frac{n s^{n-1}}{r^{n}}\left(v\left(x_{0}\right)+\frac{s^{2}}{2 n} \Delta v\left(x_{0}\right)\right) d s \\
& =v\left(x_{0}\right) \frac{n}{r^{n}} \int_{0}^{r} s^{n-1} d s+\Delta v\left(x_{0}\right) \frac{1}{2 r^{n}} \int_{0}^{r} s^{n+1} d s=v\left(x_{0}\right)+\Delta v\left(x_{0}\right) \frac{r^{2}}{2(n+2)} .
\end{aligned}
$$

Let us proceed to the proof of (A.1). Estimate (A.1) is well known for harmonic functions, which will be used to show that it also holds for biharmonic functions. We follow the proof of estimates on derivatives of harmonic functions (see for instance [5]), and employ (A.2) and (A.3). The proof uses an argument of induction on $k=|\alpha|$. The formula (A.3) implies that

$$
\left|v\left(x_{0}\right)\right| \leq \frac{1}{r^{n}\left|B_{1}\right|}\left(\|v\|_{L^{1}\left(B_{r}\left(x_{0}\right)\right)}+\frac{r^{2}}{2(n+2)}\|\Delta v\|_{L^{1}\left(B_{r}\left(x_{0}\right)\right)}\right) .
$$

Let $k=1$, then

$$
\begin{aligned}
v_{x_{i}}\left(x_{0}\right) & =f_{B_{r / 2}\left(x_{0}\right)} v_{x_{i}} d x-\frac{r^{2}}{2^{3}(n+2)} f_{B_{r / 2}\left(x_{0}\right)} \Delta v_{x_{i}} d x \\
& =\frac{2^{n}}{\left|B_{1}\right| r^{n}} \int_{\partial B_{r / 2}\left(x_{0}\right)} v v_{i} d S-\frac{r^{2} 2^{n-2}}{2(n+2)\left|B_{1}\right| r^{n}} \int_{\partial B_{r / 2}\left(x_{0}\right)} v_{i} \Delta v d S .
\end{aligned}
$$

hence

$$
\begin{aligned}
\left|v_{x_{i}}\left(x_{0}\right)\right| & \leq \frac{2 n\|v\|_{L^{\infty}\left(\partial B_{r / 2}\left(x_{0}\right)\right)}}{r}+\frac{r n\|\Delta v\|_{L^{\infty}\left(\partial B_{r / 2}\left(x_{0}\right)\right)}}{2^{2}(n+2)} \\
& \leq \frac{2^{n+1} n\|v\|_{L^{1}\left(B_{r}\left(x_{0}\right)\right)}}{\left|B_{1}\right| r^{n+1}}+\frac{n 2^{n-1} r^{2}\|\Delta v\|_{L^{1}\left(B_{r}\left(x_{0}\right)\right)}}{2(n+2)\left|B_{1}\right| r^{n+1}}+\frac{r^{2} n 2^{n-1}\|\Delta v\|_{L^{1}\left(B_{r}\left(x_{0}\right)\right)}}{2(n+2)\left|B_{1}\right| r^{n+1}} \\
& \leq \frac{n 2^{n+1}}{\left|B_{1}\right| r^{n+1}}\left(\|v\|_{L^{1}\left(B_{r}\left(x_{0}\right)\right)}+\frac{r^{2}}{2^{2}(n+2)}\|\Delta v\|_{L^{1}\left(B_{r}\left(x_{0}\right)\right)}\right) .
\end{aligned}
$$

Assuming that (A.1) is true for $k-1$, we will show that it is true for $k$. Let $|\alpha|=k$, and $D^{\alpha} v=\left(D^{\beta} v\right)_{x_{i}}$, where $|\beta|=k-1$. By (A.3)

$$
\begin{aligned}
D^{\alpha} v\left(x_{0}\right) & =f_{B_{r / k}\left(x_{0}\right)} D^{\alpha} v d x-\frac{r^{2}}{k^{2} 2(n+2)} D^{\alpha} \Delta v\left(x_{0}\right) \\
& =\frac{k^{n}}{\left|B_{1}\right| r^{n}} \int_{\partial B_{r / k}\left(x_{0}\right)} D^{\beta} v v_{i} d S-\frac{r^{2}}{k^{2} 2(n+2)} D^{\alpha} \Delta v\left(x_{0}\right)
\end{aligned}
$$

Hence

$$
\left|D^{\alpha} v\left(x_{0}\right)\right| \leq \frac{k n}{r}\left\|D^{\beta} v\right\|_{L^{\infty}\left(\partial B_{r / k\left(x_{0}\right)}\right)}+\frac{r^{2}}{k^{2} 2(n+2)}\left|D^{\alpha} \Delta v\left(x_{0}\right)\right| .
$$


If $x \in \partial B_{r / k}\left(x_{0}\right)$, then $B_{r(k-1) / k}(x) \subset B_{r}\left(x_{0}\right)$, and by induction assumption

$$
\begin{aligned}
\left|D^{\alpha} v\left(x_{0}\right)\right| \leq & \frac{k n(k-1)^{k-1}\left(2^{n+1} n\right)^{k-1} k^{n+k-1}}{r\left|B_{1}\right| r^{n+k-1}(k-1)^{n+k-1}}\|v\|_{L^{1}\left(B_{r}\left(x_{0}\right)\right)} \\
& +\frac{r^{2}(k-1)^{2} k n(k-1)^{k-1}\left(2^{n+1} n\right)^{k-1} k^{n+k-1}}{k^{2} 2(n+2) r\left|B_{1}\right| r^{n+k-1}(k-1)^{n+k-1}}\|\Delta v\|_{L^{1}\left(B_{r}\left(x_{0}\right)\right)} \\
& +\frac{r^{2}\left(2^{n+1} n k\right)^{k}}{\left|B_{1}\right| r^{n+k} k^{2} 2(n+2)}\|\Delta v\|_{L^{1}\left(B_{r}\left(x_{0}\right)\right)} \leq \frac{\left(k n 2^{n+1}\right)^{k}}{\left|B_{1}\right| r^{n+k}}\|v\|_{L^{1}\left(B_{r}\left(x_{0}\right)\right)} \\
& +\frac{\left(k n 2^{n+1}\right)^{k}\left((k-1)^{2}+1\right)}{2(n+2)\left|B_{1}\right| k^{2} r^{n+k-2}}\|\Delta v\|_{L^{1}\left(B_{r}\left(x_{0}\right)\right)} \\
\leq & \frac{\left(k n 2^{n+1}\right)^{k}}{\left|B_{1}\right| r^{n+k}}\left(\|v\|_{L^{1}\left(B_{r}\left(x_{0}\right)\right)}+\frac{r^{2}}{2(n+2)}\|\Delta v\|_{L^{1}\left(B_{r}\left(x_{0}\right)\right)}\right)
\end{aligned}
$$

and the proof of the lemma is now complete.

\section{References}

1. Frehse, J.: Zum Differenzierbarkeitsproblem bei Variationsungleichungen höherer Ordnung. Abh. Math. Sem. Univ. Hambg. 36, 140-149 (1971)

2. Frehse, J.: On the regularity of the solution of the biharmonic variational inequality. Manuscr. Math. 9, 91-103 (1973)

3. Caffarelli Luis, A., Friedman, A.: The obstacle problem for the biharmonic operator. Ann. Scuola Norm. Sup. Pisa Cl. Sci. (4) 6(1), 151-184 (1979)

4. Andersson, J.: Almost everywhere regularity for the free boundary of the normalized p-harmonic obstacle problem p $>2$. arXiv:1611.04397

5. Evans Lawrence, C.: Partial Differential Equations. Graduate Studies in Mathematics, vol. 19. American Mathematical Society, Providence, RI (2010). ISBN: 978-0-8218-4974-3

6. Friedman, A.: Variational Principles and Free-Boundary Problems. A Wiley-Interscience Publication. Pure and Applied Mathematics. Wiley, New York (1982). ISBN: 0-471-86849-3

7. Landkof, N.S.: Foundations of Modern Potential Theory. Springer, Berlin (1972)

8. Jerison David, S., Kenig, Carlos E.: Boundary behavior of harmonic functions in nontangentially accessible domains. Adv. Math. 46(1), 80-147 (1982)

9. Duffin, R.J.: Continuation of biharmonic functions by reflection. Duke Math. J. 22, 313-324 (1955)

10. Alfred, Huber: The reflection principle for polyharmonic functions. Pac. J. Math. 5, 433-439 (1955)

11. Gilbarg, D., Trudinger Neil S.: Elliptic Partial Differential Equations of Second Order. Springer, Berlin (2001). (Reprint of the 1998 edition)

Publisher's Note Springer Nature remains neutral with regard to jurisdictional claims in published maps and institutional affiliations. 